NASA Technical Paper 3568

NASA-TP-3568 NIPS-96-07316

19960010159

\title{
Buckling Behavior of Long Anisotropic Plates Subjected to Combined Loads
}

Michael P. Nemeth

\section{LIBRARY COFY}

DEC I . 31995

LANGLEY RESEARCH CENTER

LIBRARY NASA

HAMPTOU! $\mathrm{Y}$ 



\section{Buckling Behavior of Long Anisotropic Plates Subjected to Combined Loads}

Michael P. Nemeth

Langley Research Center $\bullet$ Hampton, Virginia 
Available electronically at the following URL address: http://techreports.larc.nasa.gov/ltrs/trs.html

Printed copies available from the following:

NASA Center for AeroSpace Information

800 Elkridge Landing Road

Linthicum Heights, MD 21090-2934

(301) 621-0390
National Technical Information Service (NTIS) 5285 Port Royal Road

Springfield, VA 22161-2171

(703) $487-4650$ 


\begin{abstract}
A parametric study is presented of the buckling behavior of infinitely long symmetrically laminated anisotropic plates subjected to combined loads. The study focuses on the interaction of a subcritical (stable) secondary loading state of constant magnitude and a primary destabilizing load that is increased in magnitude until buckling occurs. The loads considered in this report are uniform axial compression, pure in-plane bending, transverse tension and compression, and shear. Results are presented that were obtained by using a special purpose nondimensional analysis that is well suited for parametric studies of clamped and simply supported plates. In particular, results are presented for $a[ \pm 45]_{s}$ graphite-epoxy laminate that is highly anisotropic and representative of a laminate used for spacecraft applications. In addition, generic buckling-design charts are presented for a wide range of nondimensional parameters that are applicable to a broad class of laminate constructions. These results show the general behavioral trends of specially orthotropic plates and the effects of flexural anisotropy on plates subjected to various combined loading conditions. An important finding of the present study is that the effects of flexural anisotropy on the buckling resistance of a plate can be significantly more important for plates subjected to combined loads than for plates subjected to single-component loads.
\end{abstract}

\section{Introduction}

Buckling behavior of laminated plates is a topic of fundamental importance in the design of aerospace vehicle structures. Often the sizing of many subcomponents of these vehicles is determined by stability constraints, in addition to strength and stiffness constraints. One subcomponent that is of practical importance in structural design is the long rectangular plate. These plates commonly appear as subcomponents of stiffened panels that are used for wing structures. In addition, long plates appear as subcomponents of semimonocoque shells that are used for fuselage and launch vehicle structures. Buckling results for infinitely long plates are important because they often provide a useful conservative estimate of the behavior of finite-length rectangular plates, and they provide information that is useful in explaining the behavior of these finite-length plates. Moreover, knowledge of the behavior of infinitely long plates can provide insight into the buckling behavior of more complex structures such as stiffened panels.

An important type of long plate that appears as a subcomponent of advanced composite structures is the symmetrically laminated plate. In the present paper, the term, "symmetrically laminated plate," refers to plates in which every lamina above the plate midplane has a corresponding lamina located at the same distance below the plate midplane, with the same thickness, material properties, and fiber orientation. Symmetrically laminated plates remain flat during the manufacturing process and exhibit flat prebuckling deformation states. These characteristics and the amenability of these plates to structural tailoring provide symmetrically laminated plates with a significant potential for reducing structural weight of aircraft and launch vehicles. Thus, understanding the buckling behavior of symmetrically laminated plates is an important part of the search for ways to exploit plate orthotropy and anisotropy to reduce structural weight.

In many practical cases, symmetrically laminated plates exhibit specially orthotropic behavior. However, in some cases, such as $[ \pm 45]_{s}$ laminates, these plates exhibit anisotropy in the form of material-induced coupling between pure bending and twisting deformations. This coupling is referred to herein as flexural anisotropy, and it generally yields buckling modes that are skewed in appearance, as depicted in figure 1 . The effects of flexural orthotropy and flexural anisotropy on the buckling behavior of long rectangular plates subjected to single and combined loading conditions are becoming better understood. For example, recent in-depth parametric studies that show the effects of anisotropy on the buckling behavior of long plates subjected to compression, shear, in-plane bending, and various combinations of these loads have been presented in references 1 through 4 . The results presented in these references indicate that the importance of flexural anisotropy on the buckling resistance of long plates varies with the magnitude and type of the combined loading condition. However, the extent of the influence of the combined loading condition on the importance of neglecting flexural anisotropy in a buckling analysis is not well understood.

The objectives of the present paper are to present buckling results for specially orthotropic plates subjected to combined loads in terms of a useful nondimensional design parameter and to identify the effects of flexural 
anisotropy on the buckling behavior of long symmetrically laminated plates subjected to combined loads in a more explicit manner than has been given in references 1 and 2 . This second objective is accomplished by modeling various combined loads as a primary system of destabilizing loads with a secondary system of subcritical loads instead of using the traditional approach in which each component of a combined loading is treated as a destabilizing load with a fixed relative magnitude. This approach permits the effects of flexural orthotropy, flexural anisotropy, and combined loading characteristics on plate-buckling behavior to be obtained and presented in a direct manner. The primary destabilizing loads that are considered consist of uniform axial compression, shear, and pure in-plane bending loads; the secondary subcritical loads that are considered consist of transverse tension or compression and shear loads. Results are presented for plates with the two opposite long edges clamped or simply supported. A number of generic buckling curves that are applicable to a wide range of laminate constructions are also presented using the nondimensional parameters described in references 1,2 , and 5 .

\section{Symbols}

$A_{m}, B_{m}$

$b$

$D_{11}, D_{12}, D_{22}, D_{66} \quad$ orthotropic plate-bending

$D_{16}, D_{26}$

$E_{1}, E_{2}, G_{12}$

$K_{b} \equiv\left(n_{b 1}\right)_{c r}$

$K_{s} \equiv\left(n_{x y 1}\right)_{c r}$

$K_{x} \equiv\left(n_{x 1}^{c}\right)_{c r}$

$K_{y} \equiv\left(n_{y 1}\right)_{c r}$ stiffnesses, in-lb

displacement amplitudes (see eq. (22)), in.

plate width, (see fig. 1), in.

anisotropic plate-bending

stiffnesses, in-lb

lamina moduli, psi

nondimensional buckling coeffi-

cient associated with critical value of an eccentric in-plane bending

load (see eq. (21) and fig. 1(a))

nondimensional buckling coefficient associated with critical value of a uniform shear load (see eq. (20) and fig. 1(a))

nondimensional buckling coefficient associated with critical value of a uniform axial compression load (see eq. (18) and fig. 1(a))

nondimensional buckling coefficient associated with critical value of a uniform transverse compression load (see eq. (19) and fig. 1(a))

$$
\begin{aligned}
& K_{x} \mid \gamma=\delta=0, \\
& K_{s} \mid \gamma=\delta=0, \\
& K_{b \mid \gamma}=\delta=0
\end{aligned}
$$

$L_{1}, L_{2}, L_{3}, L_{4}$

$n_{x 1}^{c}, n_{y 1}, n_{x y 1}, n_{b 1}$

$n_{x 2}^{c}, n_{y 2}, n_{x y 2}, n_{b 2}$

$N$

$N_{b}$

$N_{x c}$

$N_{x}, N_{y}, N_{x y}$

$N_{x 1}^{c}, N_{y 1}, N_{x y 1}, N_{b 1}$

$N_{x 2}^{c}, N_{y 2}, N_{x y 2}, N_{b 2}$

$\tilde{p}, \tilde{p}_{c r}$

$w_{N}(\xi, \eta)$

$x, y$ compression, shear, and in-plane bending buckling coefficients defined by equations (18), (20), and (21), respectively, in which anisotropy is neglected in the analysis

nondimensional load factors defined by equations (14) through (17), respectively

nondimensional membrane stress resultants of system of destabilizing loads defined by equations (10) through (13), respectively

nondimensional membrane stress resultants of system of subcritical loads defined by equations (10) through (13), respectively

number of terms in series representation of out-of-plane displacement field at buckling (see eq. (22))

intensity of eccentric in-plane bending load distribution defined by equation (5), lb/in.

intensity of constant-valued tension or compression load distribution defined by equation (5), lb/in.

longitudinal, transverse, and shear membrane stress resultants, respectively (see eqs. (5), (7), and (8)), lb/in.

membrane stress resultants of system of destabilizing loads (see eqs. (6) through (9)), lb/in.

membrane stress resultants of system of subcritical loads (see eqs. (6) through (9)), lb/in.

nondimensional loading parameter (see eqs. (14) through (17)) and corresponding value at buckling (see eqs. (18) through (21)), respectively

out-of-plane displacement field at buckling defined by equation (22), in.

plate coordinate system (see fig. 1), in. 


$$
\begin{array}{ll}
\alpha_{\infty}, \beta, \gamma, \delta & \begin{array}{l}
\text { nondimensional parameters } \\
\text { defined by equations (1), (2), } \\
\text { (3), and (4), respectively }
\end{array} \\
\varepsilon_{0}, \varepsilon_{1} & \begin{array}{l}
\text { symbols that define distribution of } \\
\text { in-plane bending load (see fig. 1 } \\
\text { and eq. (5)) }
\end{array} \\
\eta=y / b, \xi=x / \lambda & \begin{array}{l}
\text { nondimensional plate coordinates } \\
\text { half-wavelength of buckling } \\
\text { mode (see fig. 1), in. }
\end{array} \\
\lambda / b & \text { buckle aspect ratio (see fig. 1) } \\
v_{12} & \begin{array}{l}
\text { lamina major Poisson's ratio } \\
\Phi_{m}(\eta)
\end{array} \\
\text { basis functions used to represent } \\
\text { buckling mode (see eq. (22)) }
\end{array}
$$

\section{Analysis Description}

Often in preparing generic design charts for buckling of a single flat plate, a special purpose analysis is preferred over a general purpose analysis code, such as a finite element code, because of the cost and effort usually involved in generating a large number of results with a general purpose code. The results presented herein were obtained using such a special purpose analysis. The analysis details are lengthy, and only a brief description of the analysis is presented.

Symmetrically laminated plates can have many different constructions because of the variety of material systems, fiber orientations, and stacking sequences that can be used to construct a laminate. A way of coping with the vast diversity of laminate constructions is to use convenient nondimensional parameters. The buckling analysis that is used in the present paper is based on classical plate theory and the classical Rayleigh-Ritz method and is derived explicitly in terms of the nondimensional parameters defined in references 1,2 , and 5 . This approach is an effective method of conducting generic in-depth parametric studies of buckling behavior in terms of the minimum number of independent parameters needed to characterize fully the buckling behavior and to obtain results that indicate overall trends and the sensitivity of the results to changes in the parameters. The nondimensional parameters used in the present paper are given by

$$
\begin{aligned}
& \alpha_{\infty}=\frac{b}{\lambda}\left(\frac{D_{11}}{D_{22}}\right)^{1 / 4} \\
& \beta=\frac{D_{12}+2 D_{66}}{\left(D_{11} D_{22}\right)^{1 / 2}}
\end{aligned}
$$

$$
\begin{aligned}
& \gamma=\frac{D_{16}}{\left(D_{11}^{3} D_{22}\right)^{1 / 4}} \\
& \delta=\frac{D_{26}}{\left(D_{11} D_{22}^{3}\right)^{1 / 4}}
\end{aligned}
$$

where $b$ is the plate width and $\lambda$ is the half-wavelength of the buckle pattern of an infinitely long plate (fig. 1). The subscripted $D$-terms are the bending stiffnesses of classical laminated plate theory. The parameters $\alpha_{\infty}$ and $\beta$ characterize the flexural orthotropy, and the parameters $\gamma$ and $\delta$ characterize the flexural anisotropy.

The loading combinations included in the analysis are uniform biaxial tension and compression, uniform shear, and eccentric in-plane bending, as depicted in figure 1 . The longitudinal stress resultant $N_{x}$ is partitioned in the analysis into a uniform tension or compression part and a linearly varying part that correspond to eccentric in-plane bending loads. This partitioning is given by

$$
N_{x}=N_{x c}-N_{b}\left[\varepsilon_{0}+\left(\varepsilon_{1}-\varepsilon_{0}\right) \eta\right]
$$

where $N_{x c}$ denotes the intensity of the constant-valued tension or compression part of the load, and the term containing $N_{b}$ defines the intensity of the eccentric inplane bending load distribution. The symbols $\varepsilon_{0}$ and $\varepsilon_{1}$ define the distribution of the in-plane bending load, and the symbol $\eta$ is the nondimensional coordinate given by $\eta=y / b$ (fig. 1).

The analysis is based on a general formulation that includes combined destabilizing loads that are proportional to a positive-valued loading parameter $\tilde{p}$ that is increased monotonically until buckling occurs and independent subcritical combined loads that remain fixed at a specified load level below the value of the buckling load. Herein, use of the term, "subcritical load," includes loads, such as a transverse tension load, that do not cause buckling to occur. In practice the subcritical loads are applied to a plate first with an intensity that is below the intensity that will cause the plate to buckle. With the subcritical loads fixed, the destabilizing loads are applied by increasing the magnitude of the loading parameter until buckling occurs. This approach permits combined-load interaction to be investigated in a direct and convenient manner.

The distinction between the destabilizing and subcritical loading systems is implemented in the buckling analysis by partitioning the prebuckling stress resultants as follows:

$$
N_{x c}=-N_{x 1}^{c}+N_{x 2}^{c}
$$




$$
\begin{gathered}
N_{y}=-N_{y 1}+N_{y 2} \\
N_{x y}=N_{x y 1}+N_{x y 2} \\
N_{b}=N_{b 1}+N_{b 2}
\end{gathered}
$$

where the stress resultants with the subscript 1 constitute the destabilizing loads, and those with the subscript 2 constitute the subcritical loads. The sign convention that is used for positive values of these stress resultants is shown in figure 1 . The normal stress resultants of the system of destabilizing loads, $N_{x 1}^{c}$ and $N_{y 1}$, are defined to be positive valued for compression loads. This convention results in positive eigenvalues being used to indicate instability caused by compression loads.

The buckling analysis includes several nondimensional stress resultants that are associated with equations (6) through (9). These dimensionless stress resultants are given by

$$
\begin{gathered}
n_{x j}^{c}=\frac{N_{x j}^{c} b^{2}}{\pi^{2}\left(D_{11} D_{22}\right)^{1 / 2}} \\
n_{y j}=\frac{N_{y j} b^{2}}{\pi^{2} D_{22}} \\
n_{x y j}=\frac{N_{x y j} b^{2}}{\pi^{2}\left(D_{11} D_{22}^{3}\right)^{1 / 4}} \\
n_{b j}=\frac{N_{b j} b^{2}}{\pi^{2}\left(D_{11} D_{22}\right)^{1 / 2}}
\end{gathered}
$$

where the subscript $j$ takes on the values of 1 and 2 . In addition, the destabilizing loads are expressed in terms of the loading parameter $\tilde{p}$ in the analysis by

$$
\begin{aligned}
& n_{x 1}^{c}=L_{1} \tilde{p} \\
& n_{y 1}=L_{2} \tilde{p} \\
& n_{x y 1}=L_{3} \tilde{p} \\
& n_{b 1}=L_{4} \tilde{p}
\end{aligned}
$$

where $L_{1}$ through $L_{4}$ are load factors that determine the specific form (relative magnitude of the load components) of a given system of destabilizing loads. Typically, the dominant load factor is assigned a value of 1 and all other factors are given as positive or negative fractions.
Nondimensional buckling coefficients are given by the values of the dimensionless stress resultants of the system of destabilizing loads at the onset of buckling; that is,

$$
\begin{gathered}
K_{x} \equiv\left(n_{x 1}^{c}\right)_{c r}=\frac{\left(N_{x 1}^{c}\right)_{c r} b^{2}}{\pi^{2}\left(D_{11} D_{22}\right)^{1 / 2}}=L_{1} \tilde{p}_{c r} \\
K_{y} \equiv\left(n_{y 1}\right)_{c r}=\frac{\left(N_{y 1}\right)_{c r} b^{2}}{\pi^{2} D_{22}}=L_{2} \tilde{p}_{c r} \\
K_{s} \equiv\left(n_{x y 1}\right)_{c r}=\frac{\left(N_{x y 1}\right)_{c r} b^{2}}{\pi^{2}\left(D_{11} D_{22}^{3}\right)^{1 / 4}}=L_{3} \tilde{p}_{c r} \\
K_{b} \equiv\left(n_{b 1}\right)_{c r}=\frac{\left(N_{b 1}\right)_{c r} b^{2}}{\pi^{2}\left(D_{11} D_{22}\right)^{1 / 2}}=L_{4} \tilde{p}_{c r}
\end{gathered}
$$

where $\tilde{p}_{c r}$ is the magnitude of the loading parameter at buckling. Positive values of the coefficients $K_{x}$ and $K_{y}$ correspond to uniform compression loads, and the coefficient $K_{s}$ corresponds to uniform positive shear. The direction of a positive shear-stress resultant acting on a plate is shown in figure 1 . The coefficient $K_{b}$ corresponds to the specific in-plane bending-load distribution that is defined by the selected values of the parameters $\varepsilon_{0}$ and $\varepsilon_{1}$.

The mathematical expression that is used in the variational analysis to represent the general off-centered and skewed buckle pattern is given by

$$
w_{N}(\xi, \eta)=\sum_{m=1}^{N}\left(A_{m} \sin \pi \xi+B_{m} \cos \pi \xi\right) \Phi_{m}(\eta)
$$

where $\xi=x / \lambda$ and $\eta=y / b$ are nondimensional coordinates, $w_{N}$ is the out-of-plane displacement field, and $A_{m}$ and $B_{m}$ are the unknown displacement amplitudes. In accordance with the Rayleigh-Ritz method, the basis functions $\Phi_{m}(\eta)$ are required to satisfy the kinematic boundary conditions on the plate edges at $\eta=0$ and $\eta=1$. For the simply supported plates the basis functions used in the analysis are given by

$$
\Phi_{m}(\eta)=\sin [m \pi \eta]
$$

for values of $m=1,2,3, \ldots, N$. Similarly, for the clamped plates, the basis functions are given by

$$
\Phi_{m}(\eta)=\cos [(m-1) \pi \eta]-\cos [(m+1) \pi \eta]
$$

Algebraic equations that govern the buckling behavior of infinitely long plates are obtained by substituting 
the series expansion for the buckling mode that is given by equation (22) into the second variation of the total potential energy and then by computing the integrals appearing in the second variation in closed form. The resulting equations constitute a generalized eigenvalue problem that depends on the aspect ratio of the buckle pattern $\lambda / b$ (fig. 1) and the nondimensional parameters and nondimensional stress resultants that are defined herein. The smallest eigenvalue of the problem corresponds to buckling and is found by specifying a value of $\lambda \mathrm{b}$ and solving the corresponding generalized eigenvalue problem for its smallest eigenvalue. This process is repeated for successive values of $\lambda b$ until the overall smallest eigenvalue is found.

Results that are obtained by using the analysis described herein have been compared with other results for isotropic, orthotropic, and anisotropic plates that have been obtained by other analysis methods. These comparisons are discussed in references 1 and 2; in every case the analysis described herein was found to be in good agreement with the results obtained from other analyses.

\section{Results and Discussion}

Results are presented for clamped and simply supported plates that are loaded by various combinations of axial compression, transverse tension or compression, pure in-plane bending, and shear. For loading cases that involve shear, a distinction is made between positive and negative shear loads whenever flexural anisotropy is present. A positive shear load corresponds to the shear loads shown in figure 1 . No distinction between positive and negative pure in-plane bending loads is necessary for flexurally anisotropic plates.

Results are presented first for $[ \pm 45]_{s}$ flexurally anisotropic plates. The importance of flexural anisotropy on the buckling behavior of a $[ \pm 45]_{s}$ plate subjected to uniform uniaxial compression, pure shear, or pure inplane bending loads is well-known and is documented in references 1 and 2 . Additional results for this laminate are presented herein that show that the importance of flexural anisotropy on the buckling behavior depends on the type of combined loading. This thin laminate is representative of spacecraft structural components and is made of a typical graphite-epoxy material with a longitudinal modulus $E_{1}=127.8 \mathrm{GPa}\left(18.5 \times 10^{6} \mathrm{psi}\right)$, a transverse modulus $E_{2}=11.0 \mathrm{GPa}\left(1.6 \times 10^{6} \mathrm{psi}\right)$, an in-plane shear modulus $G_{12}=5.7 \mathrm{GPa}\left(0.832 \times 10^{6} \mathrm{psi}\right)$, a major Poisson's ratio $v_{12}=0.35$, and a nominal ply thickness of $0.127 \mathrm{~mm}(0.005 \mathrm{in}$.). The fiber orientation angle of a lamina is measured from the plate $x$-axis to the lamina major principal axis denoted by the subscript 1 .

Generic results are presented next, in terms of the nondimensional parameters described herein, for a range of parameters that is applicable to a broad class of laminate constructions. The range of each nondimensional parameter used herein is given by $0.1 \leq \beta \leq 3.0$, $0 \leq \lambda \leq 0.6$, and $0 \leq \delta \leq 0.6$. The results presented in references 1 and 2 indicate that a value of 0.6 for $\gamma$ and $\delta$ corresponds to a highly anisotropic plate. For isotropic plates, $\beta=1$ and $\gamma=\delta=0$. Moreover, for plates without flexural anisotropy, $\gamma=\delta=0$. Values of these parameters that correspond to several practical laminates are given in references 1 and 2 .

To simplify the presentation of the fundamental generic behavioral trends, results are presented only for plates in which $\gamma$ and $\delta$ have equal values (e.g., $[ \pm 45]_{s}$ laminates). However, these behavioral trends are applicable to laminates that have nearly equal values of $\gamma$ and $\delta$ such as a $[ \pm 35 /-15]_{s}$ laminate made of the typical graphite-epoxy material described herein. For this laminate $\beta=1.95, \gamma=0.52$, and $\delta=0.51$. Furthermore, results showing the effects of $\alpha_{\infty}$, or equivalently $\left(D_{11} / D_{22}\right)^{1 / 4}$, on the buckling coefficients are not presented since it has been shown in references 1 and 2 that variations in this parameter only affect the critical value of the buckle aspect ratio $\lambda / b$ and not the buckling coefficient. (The buckling coefficient remains constant.) A value of $\left(D_{11} / D_{22}\right)^{1 / 4}=1$ was used in all the calculations presented herein. For clarity the compression, shear, and in-plane-bending buckling coefficients, defined by equations (18), (20), and (21), respectively, are expressed as $\left.K_{x}\right|_{\gamma=\delta}=0,\left.\quad K_{s}\right|_{\gamma=\delta=0}, \quad K_{b} \mid \gamma=\delta=0 \quad$ when generic results are described for which flexural anisotropy is neglected in the analysis.

\section{Importance of Anisotropy on Behavior of $[ \pm 45]_{s}$ Plates}

Results are presented in figure 2 for a clamped $[ \pm 45]_{s}$ plate subjected to a destabilizing uniform uniaxial compression load. In addition, results are presented for a combined-loading condition that consists of uniform axial compression and either a uniform subcritical transverse tension load or a compression load. Similar results are presented in figure 3 for a corresponding plate in which the destabilizing load is a uniform shear load. In these figures the minimum value of the loading parameter $\tilde{p}$ found by solving the generalized eigenvalue problem for a given value of $\lambda / b$ is shown for values of $0 \leq \lambda / b \leq 2$. Moreover, the magnitude of the subcritical transverse load is indicated in the figures by the value of the nondimensional stress resultant $n_{y 2}$ that is defined by equation (11). A limiting value of the subcritical load given by $n_{y 2}=-4$ corresponds to a widecolumn buckling mode. The dashed lines shown in the figures correspond to the solutions of the generalized eigenvalue problem that are obtained when flexural anisotropy is included in the analysis. In contrast, the 
solid lines correspond to the solutions that are obtained when flexural anisotropy is neglected in the analysis (i.e., $D_{16}=D_{26}=0$ ). The overall minimum value of the loading parameter for each curve corresponding to $n_{y 2}=0$ is indicated by an unfilled circle, and the minimum for each curve that corresponds to nonzero values of $n_{y 2}$ is indicated by a filled circle. These minimum values of the loading parameter correspond to the value of the buckling coefficient for each curve. The corresponding values of $\lambda \mathrm{b}$ are the critical values of the buckle aspect ratio.

The results presented in figure 2 indicate that the buckling coefficient (minimum loading parameter) of a plate that is loaded by pure compression only $\left(n_{y 2}=0\right)$ is overestimated by approximately 30 percent of the anisotropic buckling load and that the critical value of the buckle aspect ratio $\lambda / b$ is slightly overestimated if the flexural anisotropy is neglected in the buckling analysis. For a subcritical transverse tension load given by $n_{y 2}=3.5$ (87.5 percent of the magnitude for a widecolumn buckling mode), the results show that the effect of the flexural anisotropy becomes slightly less important; that is, the buckling coefficient is overestimated by 22 percent for this case. Neglecting the anisotropy for this case also yields results that slightly overestimate the critical value of the buckle aspect ratio. However, for a subcritical transverse compression load given by $n_{y 2}=-3.5$, the buckling coefficient is overestimated by 76 percent when the flexural anisotropy is neglected in the analysis, and the critical value of the buckle aspect ratio is slightly underestimated.

The results shown in figure 3 for a shear-loaded plate with a subcritical transverse tension or compression load and the results shown in figure 2 indicate that the effect of neglecting the flexural anisotropy in the buckling analysis is much more pronounced for the shear-loaded plate than for the corresponding compression-loaded plate. In particular, the results presented in figure 3 indicate that results that are obtained by neglecting the flexural anisotropy in the buckling analysis of a plate that is loaded by shear only $\left(n_{y 2}=0\right)$ overestimate the buckling coefficient by approximately 97 percent of the anisotropic buckling load compared with 30 percent for the corresponding compression-loaded plate. For a subcritical transverse tension load given by $n_{y 2}=3.5$, the results for the shearloaded plate predict that the buckling coefficient is overestimated by approximately 75 percent if flexural anisotropy is neglected. More significantly, for a subcritical transverse compression load given by $n_{y 2}=-3.5$, the results predict that the shear buckling coefficient is overestimated by approximately 209 percent when the flexural anisotropy is neglected in the analysis. For each subcritical loading case, the critical values of the buckle aspect ratio are overestimated when the anisotropy is neglected in the buckling analysis.
Results that are similar to the results presented in figures 2 and 3 are presented in figures 4 and 5 for a clamped $[ \pm 45]_{s}$ plate that is loaded by a subcritical shear load and either a destabilizing uniform axial compression load or a pure in-plane bending load, respectively. Two groups of curves are shown in the figures that correspond to plates without a subcritical load and with either a positive or negative subcritical shear load with a magnitude equal to 75 percent of the corresponding shear buckling coefficient $K_{s}$ (eq. (20)). For the cases in which the anisotropy is included in the analysis, the buckling coefficients for positive and negative shear loads are given by $K_{s}=6.12$ and $K_{s}=-17.16$, respectively. For the cases in which the anisotropy is neglected, the buckling coefficients for positive and negative shear loads are given by $K_{s}=12.03$ and $K_{s}=-12.03$, respectively. The results shown in figures 4 and 5 therefore include the effects of neglecting the anisotropy in the calculation of the subcritical load $n_{x y 2}$ and the actual calculation of the buckling coefficient.

The results shown in figures 4 and 5 indicate that neglecting the flexural anisotropy in a buckling analysis of the plate that is subjected only to uniform axial compression or pure in-plane bending $\left(n_{x y 2}=0\right)$ yields a solution that overestimates the buckling coefficient by approximately 30 percent of the anisotropic buckling load, and the critical value of the buckle aspect ratio is slightly overestimated. For the subcritical positive shear load with $n_{x y 2}=0.75 K_{s}$, the results predict that the buckling coefficient is overestimated by approximately 84 percent and 66 percent of the anisotropic buckling coefficient for the uniform axial compression loads and the pure in-plane bending loads, respectively. Moreover, for both loading conditions, the critical value of the buckle aspect ratio is also slightly overestimated when the anisotropy is neglected in the analysis. In contrast, for the subcritical negative shear load with $n_{x y 2}=0.75 K_{s}$, the results predict that the buckling coefficient is underestimated by approximately 26 percent and 16 percent of the anisotropic buckling coefficient for the uniform axial compression and pure in-plane bending loading conditions, respectively. In addition, the critical value of the buckle aspect ratio is slightly underestimated for the uniform axial compression load and slightly overestimated for the pure in-plane bending load when the flexural anisotropy is neglected in the analysis.

\section{Generic Results for Specially Orthotropic Plates}

A broad range of buckling results for specially orthotropic plates that are subjected to combined loads and that have either clamped or simply supported edges are presented in this section. These results are included to demonstrate the effects of flexural orthotropy on platebuckling behavior in a general manner and to provide 
design curves that can be used in conjunction with the results presented in the next section to obtain buckling coefficients of flexurally anisotropic plates.

Results are presented in figures 6 through 10 that show the generic effects of plate flexural orthotropy on the buckling coefficients of clamped and simply supported plates with $\gamma=\delta=0$. The results in figures 6 through 8 are for plates that are subjected to a subcritical transverse tension or compression load and a destabilizing uniform axial compression load, pure in-plane bending load, and shear load, respectively. Similarly, the generic effects of flexural orthotropy on plates that are subjected to a subcritical shear load and a uniform axial compression load or to a pure in-plane bending load are shown in figures 9 and 10, respectively. The results in figures 9 and 10 are also applicable to plates that are loaded in negative shear since the shear buckling coefficients for specially orthotropic plates that are loaded in positive or negative shear have the same magnitude. Two sets of curves are shown in figures 6 through 10 for values of the orthotropic parameter $\beta=0.5,1,1.5,2,2.5$, and 3 . The solid curves correspond to results for clamped plates and the dashed curves are for simply supported plates. These curves show the buckling coefficient as a function of the nondimensional transverse load $n_{y 2}$ in figures 6 through 8 and as a function of the nondimensional shear load $n_{x y 2}$ in figures 9 and 10 .

The results presented in figures 6 through 10 indicate that the buckling coefficients of specially orthotropic plates $(\gamma=\delta=0)$ increase substantially as the orthotropic parameter $\beta$ increases. Furthermore, the results in figures 6 through 8 indicate that as the subcritical transverse load increases through positive values (increasing tension), the buckling coefficients increase substantially. This trend is shown to be more pronounced for the shearloaded plates than for the compression-loaded plates or for the plates that are loaded by pure in-plane bending. Moreover, the increase in buckling coefficient with increasing subcritical tension load is predicted to be slightly more pronounced for the simply supported plates than for the clamped plates. The results presented in figures 9 and 10 for the plates with subcritical shear loads indicate that the buckling coefficient is substantially reduced as the magnitude of the nondimensional shear load increases. In addition, the results also predict this trend to be slightly more pronounced for the simply supported plates than for the clamped plates.

\section{Interaction of Flexural Anisotropy and Loading}

Results are presented in this section that show the generic effects of plate flexural anisotropy and combined loading condition on the buckling coefficients of clamped and simply supported plates. First, results are presented for plates that are subjected to a subcritical transverse tension or compression load and destabilizing uniform axial compression, pure in-plane bending, and shear loads, respectively. Then, results are presented for plates subjected to a subcritical shear load and to destabilizing uniform uniaxial compression and pure in-plane bending loads, respectively.

Plates subjected to subcritical transverse tension or compression loads. Results are presented in figures 11 and 12,13 and 14 , and 15 and 16, respectively, for plates subjected to a subcritical transverse tension or compression load and destabilizing uniform axial compression, pure in-plane bending, and shear loads. In figures 11 through 16, the ratio of the anisotropic buckling coefficient to the corresponding orthotropic buckling coefficient that is computed with $\gamma=\delta=0$ (figs. 6 through 10) is given as a function of the orthotropic parameter $\beta$ for equal values of the anisotropic parameters $(\gamma=\delta)$ ranging from 0.1 to 0.6 . For each value of $\gamma=\delta$ that is given in figures 11 through 16, three curves are presented. The solid lines correspond to a value of the nondimensional transverse load $n_{y 2}=0$ that is used in the calculations (no subcritical load). Similarly, the finely dashed and coarsely dashed lines correspond to values of $n_{y 2}=-0.5$ (compression) and 0.5 (tension), respectively, for the simply supported plates, and to values of $n_{y 2}=-2$ (compression) and 2 (tension), respectively, for the clamped plates. The magnitudes of these values correspond to 50 percent of $K_{y}$, the buckling coefficient that corresponds to a wide-column buckling mode ( $K_{y}=1$ and 4 for simply supported and clamped plates, respectively).

The results that are presented in figures 11 and 12 , respectively, for simply supported and clamped plates subjected to a subcritical transverse tension or compression load and to destabilizing uniform uniaxial compression show that the anisotropic buckling coefficient is always less than the corresponding orthotropic buckling coefficient for the full range of parameters considered. In addition, these results predict that the effects of neglecting anisotropy are more pronounced for the plates with the transverse compression loads than for the plates without a subcritical load $\left(n_{y 2}=0\right)$ or a transverse tension load. Moreover, these results predict that this trend is slightly more pronounced for the clamped plates than for the simply supported plates, as indicated by the increase in the relative spacing of the three curves that correspond to each fixed value of $\gamma$ and $\delta$. The results also predict that the reduction in buckling coefficient caused by anisotropy is generally larger for the clamped plates with $n_{y 2}=-2$ (transverse compression at a magnitude of 50 percent of the wide-column buckling coefficient) than for the corresponding simply supported plates. This trend is indicated by the observation that the curve for the 
clamped plates generally lies below the corresponding curve for the simply supported plates for each fixed value of $\gamma$ and $\delta$. Based on a similar observation, the results also predict, however, that the reduction in buckling coefficient caused by anisotropy is generally smaller for the clamped plates with $n_{y 2}=0$ or 2 (transverse tension at a magnitude of 50 percent of the wide-column buckling coefficient) than for the corresponding simply supported plates.

The results presented in figures 13 and 14 for simply supported and clamped plates, respectively, that are subjected to a subcritical transverse tension or compression load and a destabilizing pure in-plane bending load, also show that the anisotropic buckling coefficient is always less than the corresponding orthotropic buckling coefficient for the full range of parameters being considered. These results also predict that the effects of neglecting anisotropy are more pronounced for plates with a transverse compression load than for plates with $n_{y 2}=0$ or the transverse tension load, but not to the extent that is exhibited by the corresponding plates with the uniaxial compression load. (Compare the difference in the relative spacing of the three curves that correspond to each fixed value of $\gamma$ and $\delta$ that is shown in figures 11 through 14.) Moreover, these results predict that this trend is slightly more pronounced for clamped plates than for simply supported plates, but also not to the extent that is predicted for the corresponding plates with a uniaxial compression load. Furthermore, like the plates with a uniaxial compression load, the results also predict that the reduction in buckling coefficient caused by anisotropy is generally larger for clamped plates with $n_{y 2}=-2$ (transverse compression) than for the corresponding simply supported plates. Similarly, the results also predict that the reduction in buckling coefficient caused by anisotropy is generally smaller for clamped plates with $n_{y 2}=0$ or 2 (transverse tension) than it is for the corresponding simply supported plates.

The results for simply supported plates subjected to a subcritical transverse tension or compression load and a positive destabilizing shear load presented in figure 15 and corresponding results for clamped plates presented in figure 16 also show that the anisotropic buckling coefficient is always less than the corresponding orthotropic buckling coefficient. However, these results also show that this trend is reversed for negative shear loads. These results also predict that the effects of neglecting anisotropy are generally more pronounced for plates with transverse compression loads than they are for plates with $n_{y 2}=0$ or with transverse tension loads. This trend for the shear-loaded plates is slightly less pronounced than it is for the corresponding plates with the destabilizing uniaxial compression loads and slightly more pronounced for the corresponding plates with the desta- bilizing pure in-plane bending loads. Moreover, these results predict that this trend is also slightly more pronounced for clamped plates than for simply supported plates. Furthermore, like the plates with the uniaxial compression loads, the results predict that the reduction in buckling coefficient caused by anisotropy is generally larger for clamped plates loaded in positive shear and with $n_{y 2}=-2$ (transverse compression) than for the corresponding simply supported plates. Similarly, the results also predict that the reduction in buckling coefficient caused by anisotropy is generally smaller for the clamped plates loaded in positive shear and with $n_{y 2}=0$ or 2 (transverse tension) than for the corresponding simply supported plates. For the plates loaded by negative shear, these trends are reversed. Overall, the results presented in figures 11 through 16 indicate that plates loaded by positive shear typically exhibit substantially larger reductions in the buckling coefficient when flexural anisotropy is included in the analysis than do the corresponding plates loaded by uniform axial compression or pure in-plane bending. In contrast, the results for plates that are loaded by negative shear predict sizable increases in the buckling coefficients when the flexural anisotropy is included in the calculations.

Plates subjected to subcritical shear load. Results are presented in figures 17 through 20 and in figures 21 through 24 for plates that are subjected to a subcritical shear load and to destabilizing uniform axial compression and pure in-plane bending, respectively. Moreover, the results in figures $17,18,21$, and 22 are for a positive subcritical shear load, and the results in figures 19, 20, 23 , and 24 are for a negative subcritical shear load. In figures 17 through 24, the ratio of the anisotropic buckling coefficient to the corresponding orthotropic buckling coefficient computed with $\gamma=\delta=0$ (figs. 6 through 10) is given as a function of the orthotropic parameter $\beta$ for equal values of the anisotropic parameters $(\gamma=\delta)$ ranging from 0.1 to 0.6 . For each value of $\gamma=\delta$ that is given in figures 17 through 24 , four curves are presented. The solid lines correspond to the value of the nondimensional shear load $n_{x y 2}=0$ (no subcritical load). In addition, the finely dashed, moderately dashed, and coarsely dashed lines correspond to values of $n_{x y 2}=0.25 K_{s}, 0.5 K_{s}$, and $0.8 K_{s}$, respectively, where $K_{s}$ is the shear buckling coefficient. More specifically, $K_{s}$ is the positive shear buckling coefficient in figures $17,18,21$, and 22 , and the negative shear buckling coefficient in figures 19, 20, 23, and 24 . For each case, the shear buckling coefficient is a function of $\beta, \gamma$, and $\delta$.

Points on the curves shown in figures 17 through 24 are obtained by calculating the shear buckling coefficient first, assigning the appropriate subcritical value of $n_{x y 2}$, and then computing the buckling coefficient of the 
system of destabilizing loads. Since the calculation of the shear buckling coefficient that is used in specifying the subcritical loading magnitude also depends on $\gamma$ and $\delta$, the results shown in figures 17 through 24 represent the overall effect of neglecting flexural anisotropy in both the calculation of the subcritical shear loading magnitude and the buckling coefficient of the system of destabilizing loads. This computational procedure is described subsequently in detail by using the results shown in figures 25 and 26 for the case of $n_{x y 2}=0.8 K_{s}$ and for plates with $\beta=3$ that are subjected to a subcritical shear load and to uniform uniaxial compression and pure in-plane bending, respectively.

Buckling interaction curves for plates that are subjected to a subcritical shear load and uniform uniaxial compression and to a subcritical shear load and pure inplane bending are shown in figures 25 and 26, respectively. The solid curve shown in each figure corresponds to results for specially orthotropic plates with $\gamma=\delta=0$, and the dashed lines correspond to results for $\gamma=\delta=0.6$. Furthermore, the unfilled and filled circles on the curves correspond to results for $n_{x y 2}=0.8 K_{s}$ and for positive and negative shear loading, respectively. The ordinates of the filled and unfilled circles on the curves for $\gamma=\delta=0$ correspond to the buckling coefficients $\left.K_{x}\right|_{\gamma=\delta}=0$ and $\left.K_{b}\right|_{\gamma=\delta=0}$ that are indicated in figures 17 through 20 and in figures 21 through 24 , respectively. Similarly, the ordinates of the filled and unfilled circles on the curves for $\gamma=\delta=0.6$ correspond to the buckling coefficients $K_{x}$ and $K_{b}$ that are also indicated in figures 17 through 20 and in fig-ures 21 through 24 , respectively. The buckling coefficient ratios shown in figures 17 through 24 for the case $n_{x y 2}=0.8 K_{s}$ are obtained by dividing the value of the ordinate on the curve for $\gamma=\delta=0.6$ (in figs. 25 and 26) by the corresponding value of the ordinate for the curve for $\gamma=\delta=0$ for either the filled or unfilled circles. For positive subcritical shear loading, the results in figures 25 and 26 indicate that the buckling coefficient ratios for plates subjected to uniform uniaxial compression and pure inplane bending have values less than 1 . In contrast, for negative shear loading, the results in figures 25 and 26 indicate that the buckling coefficient ratios have values greater than 1 . This trend reversal is caused by the horizontal shift in the buckling interaction curves that occurs when anisotropy is present.

The results presented in figures 17 and 18 for simply supported and clamped plates, respectively, loaded by uniform axial compression and a positive subcritical shear load, predict monotonic reductions in buckling coefficient with increasing values of the anisotropic parameters. This same trend is also predicted for increasing magnitudes of the positive shear load as indicated by the four different line types shown in figures 17 and 18.
Moreover, these results show that the reductions in buckling coefficients are generally more pronounced for the simply supported plates than for the clamped plates. This trend is also indicated by the observation that each curve for the clamped plates generally lies above the corresponding curve for the simply supported plates for a fixed value of $\gamma$ and $\delta$. More importantly, these results indicate that the reduction in buckling coefficient that is associated with including anisotropy in the calculations increases substantially as the magnitude of the positive subcritical shear load increases.

The results shown in figures 19 and 20 for simply supported and clamped plates, respectively, which are loaded by destabilizing uniform axial compression and a negative subcritical shear load, predict monotonic reductions in buckling coefficient with increasing values of the anisotropic parameters for the values of $n_{x y 2}=0$ and $0.25 K_{s}$. However, for the larger values of $n_{x y 2}$ shown in the figures, the results predict increases in buckling coefficient with increasing values of the anisotropic parameters, as anticipated from the previous results that have been presented. This seemingly unusual trend for the negative shear load is a manifestation of the horizontal shift in the compression-shear buckling interaction curves caused by flexural anisotropy that has been reported in reference 1 and illustrated in figure 25 . In addition, these results show this trend of increasing buckling coefficient with increasing values of the anisotropic parameters to be, overall, slightly more pronounced for clamped plates than for simply supported plates. The trend of a decreasing buckling coefficient with decreasing values of the anisotropic parameters is predicted, overall, to be slightly more pronounced for simply supported plates than for clamped plates. Furthermore, these results indicate that the importance of anisotropy on the buckling resistance changes dramatically with the magnitude of a negative subcritical shear load.

Results similar to those presented in figures 17 and 18 are presented in figures 21 and 22 for simply supported and clamped plates, respectively, that are loaded by pure in-plane bending and a positive subcritical shear load. These results also predict monotonic reductions in buckling coefficient with increasing values of the anisotropic parameters and increasing magnitudes of a positive subcritical shear load. However, these results show that the reductions in buckling coefficient are practically the same for the simply supported and clamped plates. The results also indicate that the reduction in buckling coefficient associated with having anisotropy included in the calculations increases substantially as the magnitude of the positive subcritical shear load increases. Comparing the results in figures $17,18,21$, and 22 suggests that the reductions in buckling coefficient that are caused by including flexural anisotropy in the analysis 
are nearly the same for the plates loaded by uniform axial compression and pure in-plane bending with $n_{x y 2}=0$. However, the comparison also suggests that the reductions in buckling coefficient that are caused by including flexural anisotropy in the analysis are more pronounced for plates that are loaded by uniform axial compression than for those that are loaded by pure in-plane bending when a nonzero subcritical shear loading is present, especially for the larger magnitudes of a subcritical shear load. This trend is also indicated by the observation that each curve for the compression-loaded plates generally lies below the corresponding curve for the plates that are subjected to pure in-plane bending, for a fixed value of $\gamma$ and $\delta$.

Results similar to those presented in figures 19 and 20 are shown in figures 23 and 24 for simply supported and clamped plates, respectively, that are loaded by pure in-plane bending and a negative subcritical shear load. These results also predict monotonic reductions in buckling coefficient with increasing values of the anisotropic parameters for the values of $n_{x y 2}=0$ and $0.25 K_{s}$. Unlike the results for compression-loaded plates, the results in figures 23 and 24 predict monotonic reductions in buckling coefficient with increasing values of the anisotropic parameters for $n_{x y 2}=0.5 K_{s}$. For $n_{x y 2}=0.8 K_{s}$, the results predict increases in buckling coefficient with increasing values of the anisotropic parameters. This unusual trend for a negative shear load is also a manifestation of the horizontal shift in the pure in-plane bending-shear buckling interaction curves caused by flexural anisotropy that has been reported in reference 2 and illustrated in figure 26. Moreover, the difference between the trends for plates that are loaded by negative subcritical shear and pure in-plane bending and by negative subcritical shear and uniform axial compression is manifested by the pronounced difference in the shape of the corresponding buckling interaction curves shown in figures 25 and 26. (See also refs. 1 and 2.) For the curves in figures 23 and 24 that show increases in buckling coefficient with increasing values of the anisotropic parameters, the results predict that the increases in buckling coefficient are generally more pronounced for the clamped plates than for the simply supported plates. In contrast, for the curves that show decreases in buckling coefficient with increasing values of the anisotropic parameters, the results predict that the decreases in buckling coefficient are slightly more pronounced for the simply supported plates than for the clamped plates. Comparing the results in figures 19, 20, 23, and 24 indicates that the curves in the figures, which show increases in buckling coefficient with increasing values of the anisotropic parameters, also show that the increases in buckling coefficient are, overall, much more pronounced for the compression-loaded plates than for the plates loaded by pure in-plane bending. Moreover, the results indicate that the curves for plates with nonzero subcritical shear loads, which show decreases in buckling coefficient with increasing values of the anisotropic parameters, also show that the decreases in buckling coefficient are, overall, much more pronounced for the plates loaded by pure in-plane bending than for the compression-loaded plates.

\section{Concluding Remarks}

A parametric study has been presented of the buckling behavior of infinitely long symmetrically laminated anisotropic plates subjected to combined loads. A special purpose nondimensional analysis that is well suited for parametric studies of clamped and simply supported plates has been described, and its key features have been discussed. The results presented herein have focused on the interaction of a subcritical (stable) secondary loading state and a primary destabilizing loading state. The interaction of uniform axial compression, pure in-plane bending, transverse tension and compression, and shear loads with plate flexural anisotropy and orthotropy have been examined. In particular, results have been presented for $[ \pm 45]_{s}$ thin graphite-epoxy laminates that are representative of spacecraft structural components. In addition, a number of generic buckling results have been presented that are applicable to a broad class of laminate constructions and show explicitly the effects of flexural orthotropy and flexural anisotropy on plate-buckling behavior under combined loads.

The most significant finding of the present study is that the importance of flexural anisotropy on the buckling behavior of a long plate is strongly dependent on the type and magnitude of the combined load that is applied. Specifically, the results presented show that significant errors (on the order of 100 percent) can be made when calculating buckling coefficients of plates subjected to combined loads if flexural anisotropy is neglected. Overall, the results presented herein show that the buckling coefficients increase significantly as the orthotropic parameter $\beta=\frac{D_{12}+2 D_{66}}{\left(D_{11} D_{22}\right)^{1 / 2}}$ increases, and that they decrease significantly as the anisotropic parameters $\gamma=\frac{D_{16}}{\left(D_{11}^{3} D_{22}\right)^{1 / 4}}$ and $\delta=\frac{D_{26}}{\left(D_{11} D_{22}^{3}\right)^{1 / 4}}$ with equal values $(\gamma=\delta)$ increase for all load combinations that are considered, except those involving negative shear loads. For the negative shear loading condition, the trend is generally reversed. The results that are presented herein also show, in many cases, that the effects of plate anisotropy are more pronounced for clamped plates than for 
simply supported plates that are subjected to combined loads.

\section{NASA Langley Research Center}

Hampton, VA 23681-0001

September 22, 1995

\section{References}

1. Nemeth, Michael P.: Buckling Behavior of Long Symmetrically Laminated Plates Subjected to Combined Loadings. NASA TP-3195, May 1992.

2. Nemeth, M. P.: Buckling of Symmetrically Laminated Plates With Compression, Shear, and In-Plane Bending. AIAA J., vol. 30, no. 12, Dec. 1992, pp. 2959-2965.
3. Zeggane, Madjid; and Sridharan, Srinivasan: Buckling Under Combined Loading of Shear Deformable Laminated Anisotropic Plates Using "Infinite" Strips. Int. J. Num. Meth. Eng., vol. 31, May 1991, pp. 1319-1331.

4. Zeggane, M.; and Sridharan, S.: Stability Analysis of Long Laminate Composite Plates Using Reissner-Mindlin Infinite Strips. Comp. \& Struc., vol. 40, no. 4, 1991, pp. 1033-1042.

5. Nemeth, Michael P.: Importance of Anisotropy on Buckling of Compression-Loaded Symmetric Composite Plates. AIAA J. vol. 24, Nov. 1986, pp. 1831-1835. 


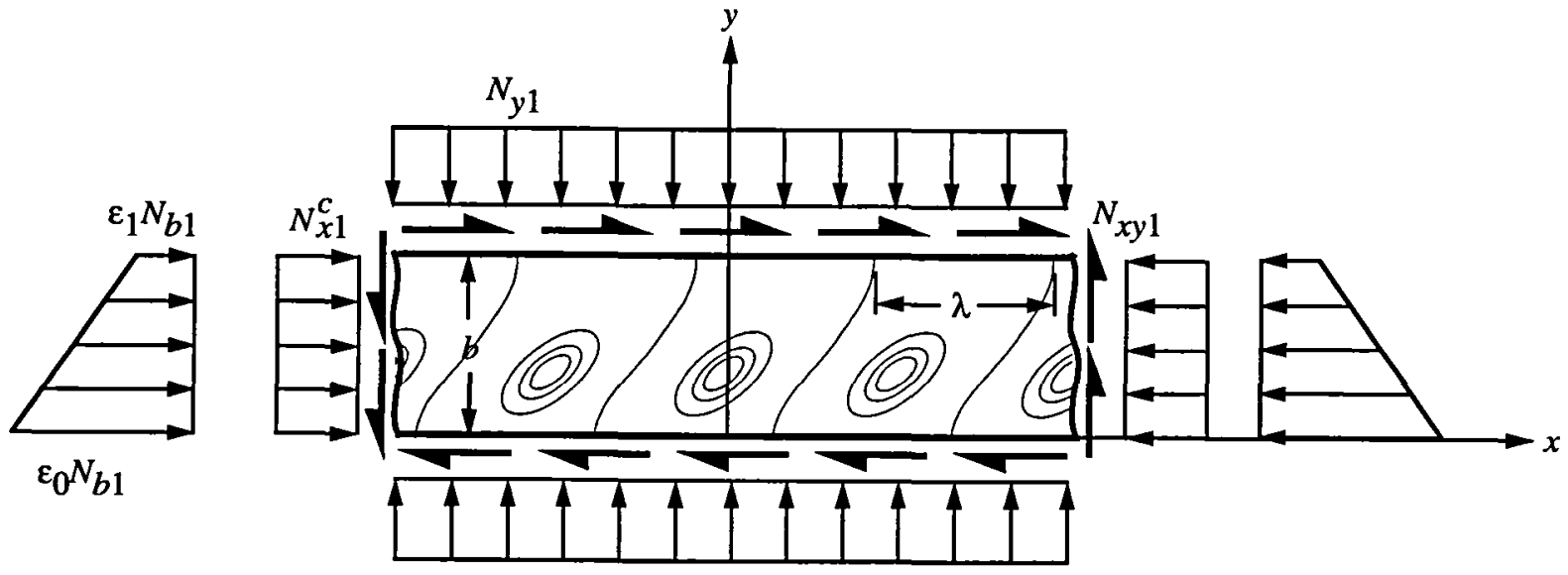

(a) Primary (destabilizing) loading system.

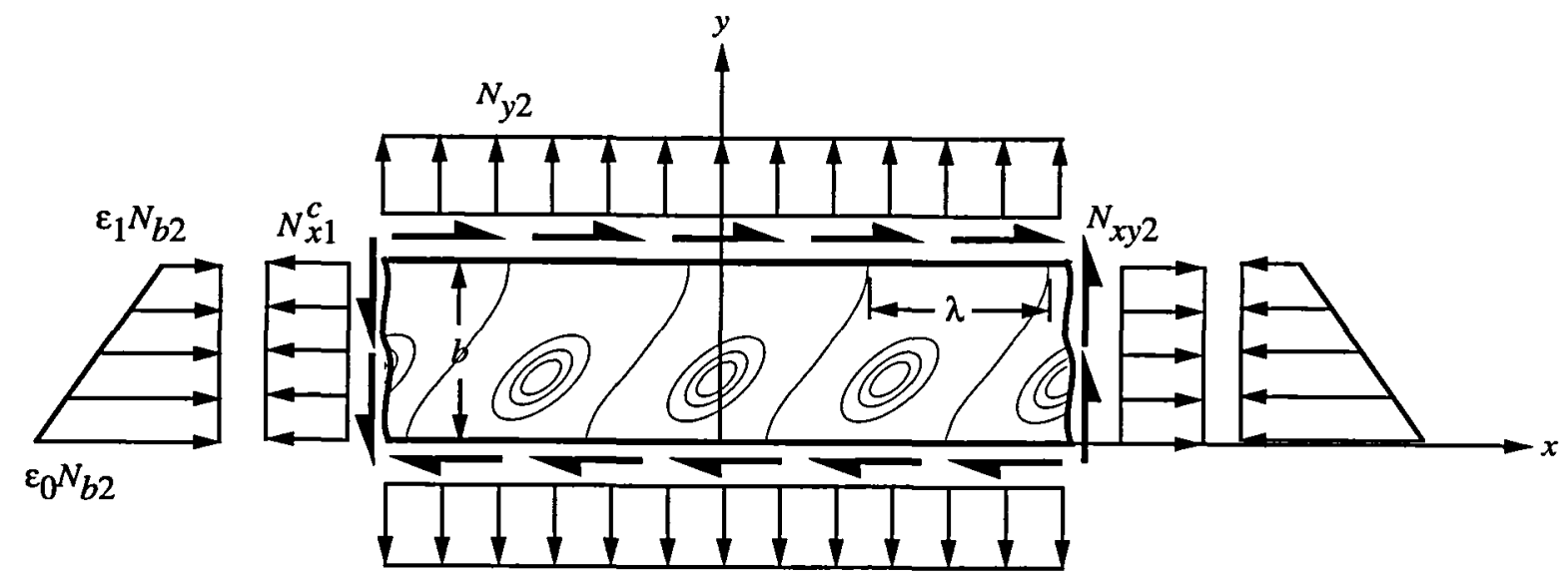

(b) Secondary (subcritical) loading system.

Figure 1. Sign convention for positive-valued stress resultants $\left(\varepsilon_{0}>\varepsilon_{1}>0\right.$ shown above). 


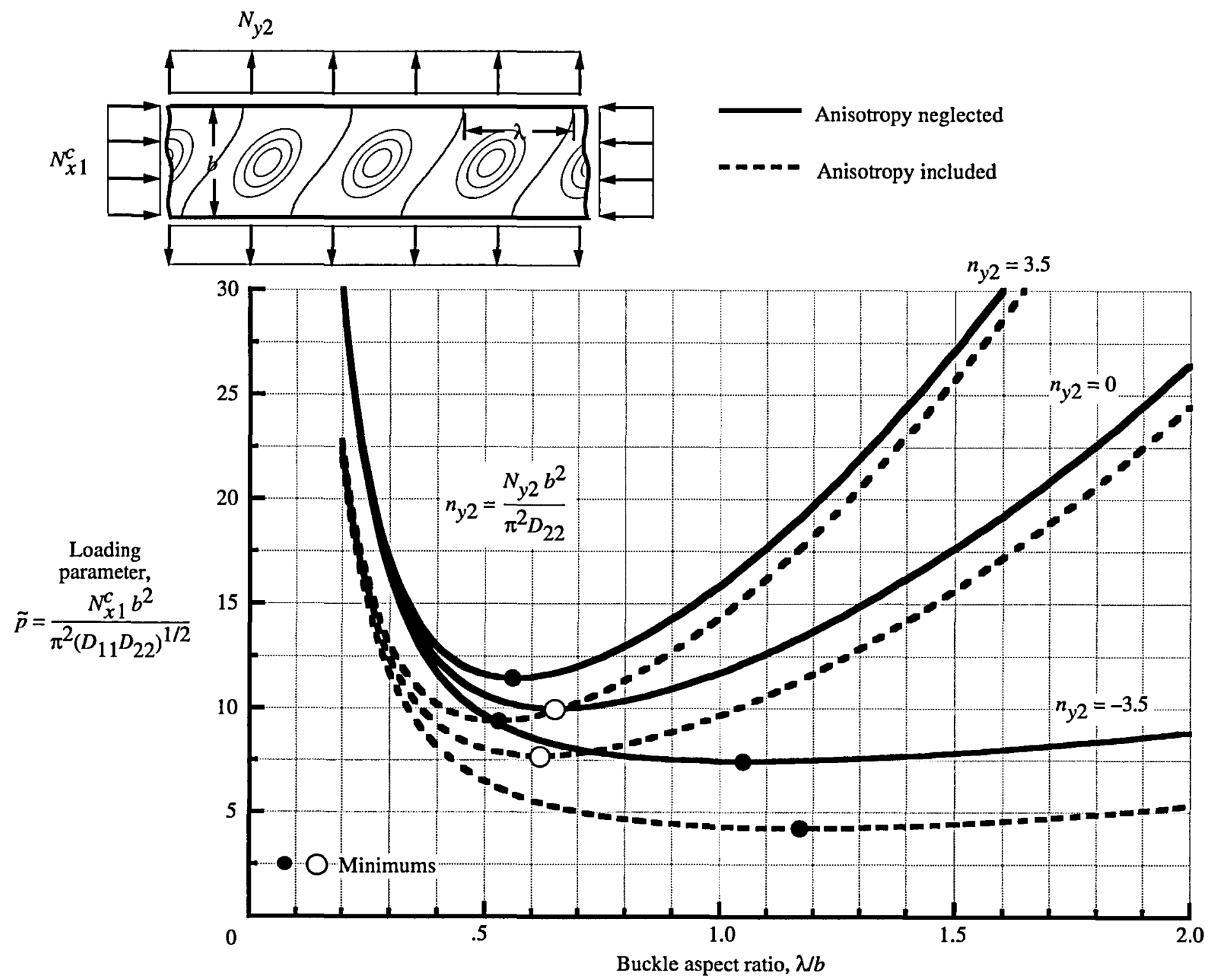

Figure 2. Buckling results for clamped $[ \pm 45]_{s}$ plates subjected to axial compression and subcritical transverse tension or compression loads. 


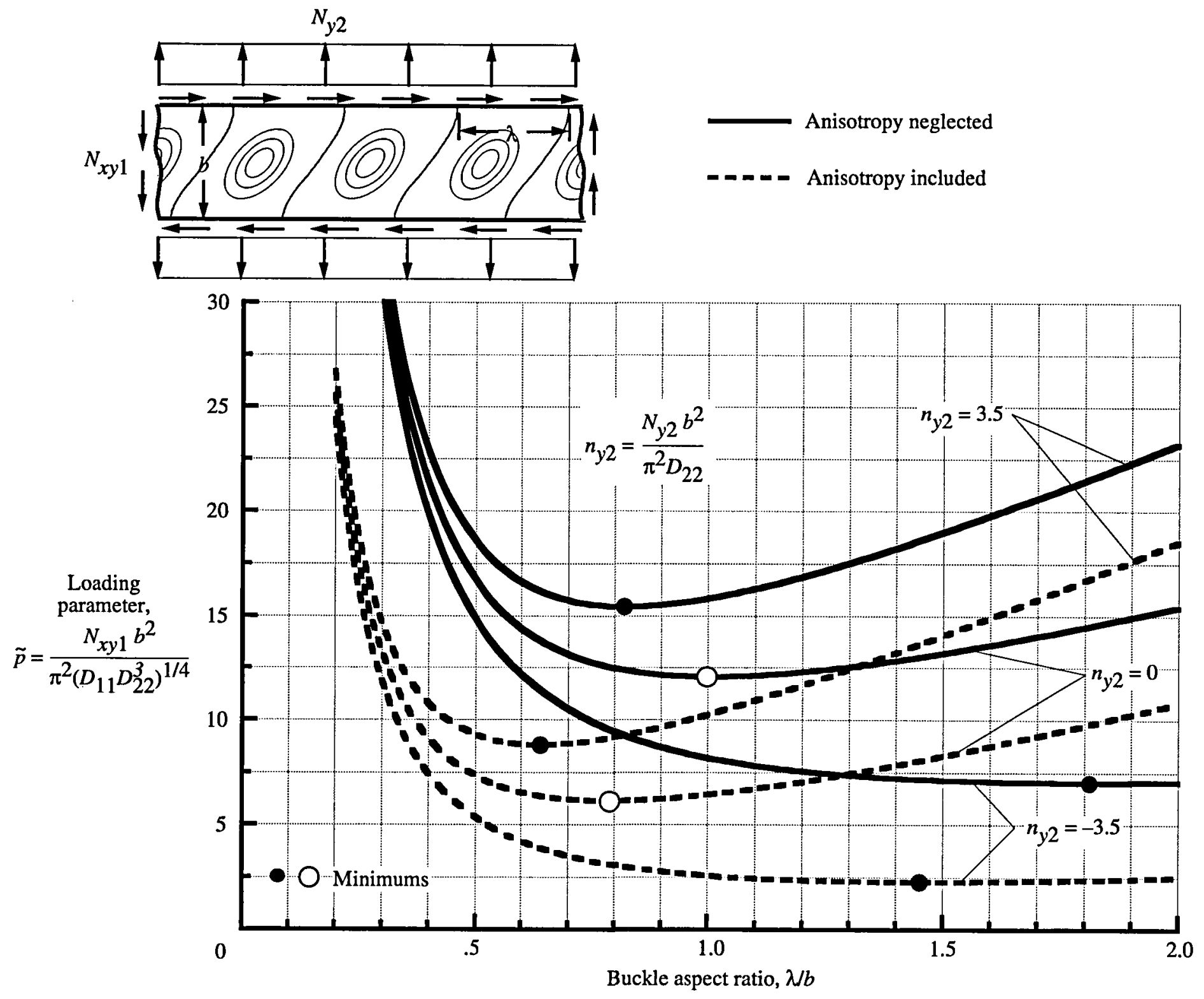

Figure 3. Buckling results for clamped $[ \pm 45]_{s}$ plates subjected to shear and subcritical transverse tension or compression loads. 

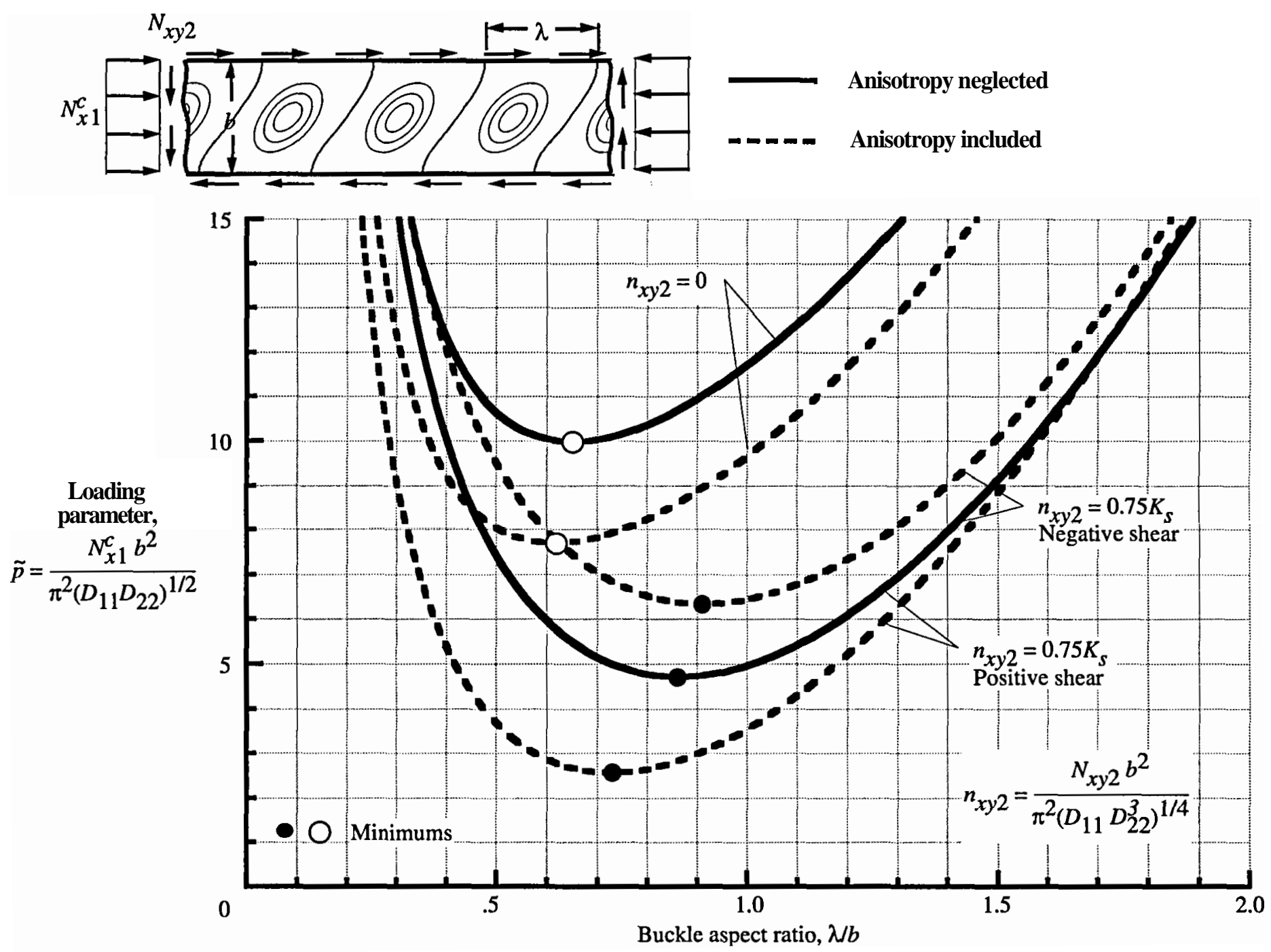

Figure 4. Buckling results for clamped $[ \pm 45]_{s}$ plates subjected to axial compression and subcritical shear loads. 


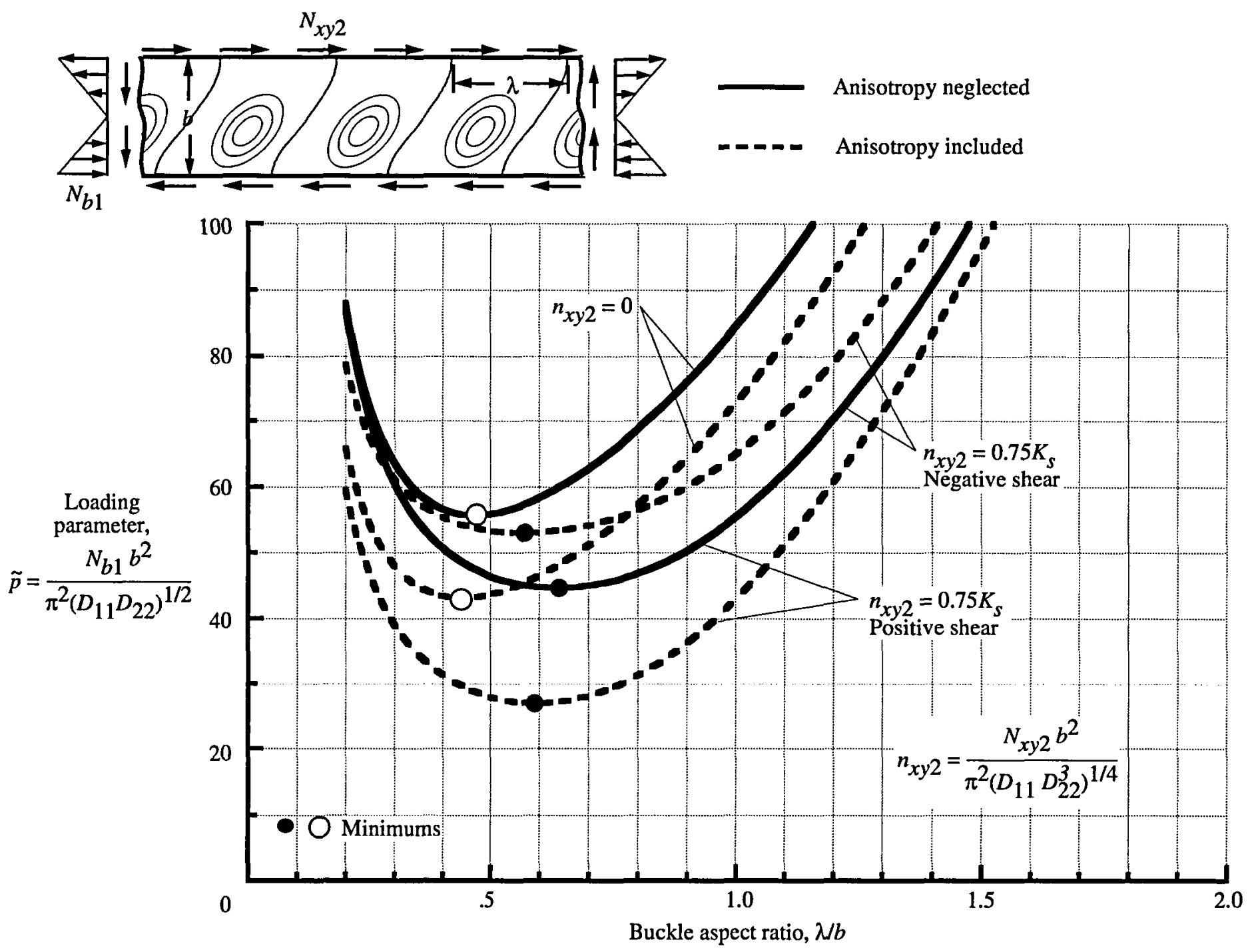

Figure 5. Buckling results for clamped $[ \pm 45]_{s}$ plates subjected to pure in-plane bending and subcritical shear loads. 


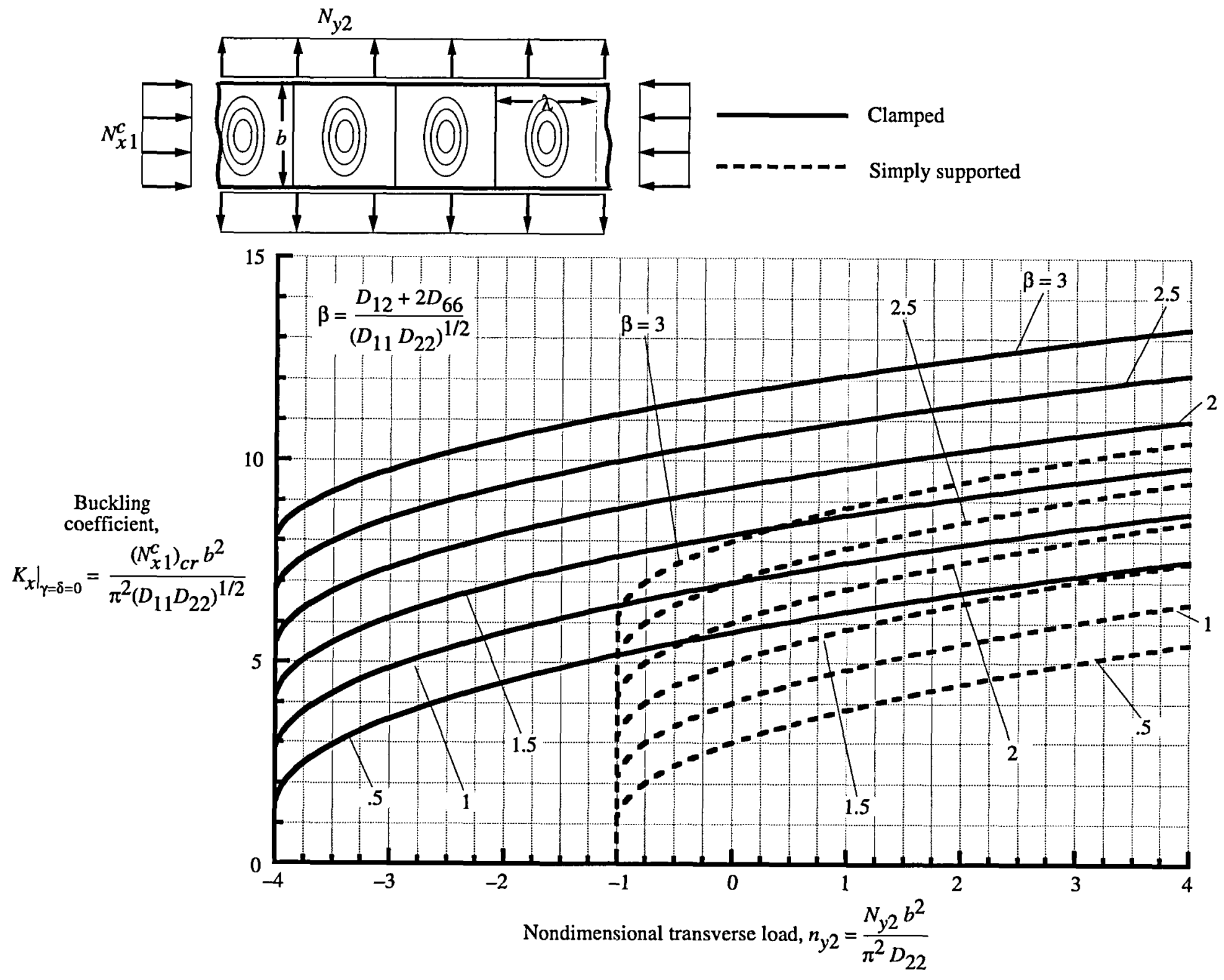

Figure 6. Effects of orthotropic parameter $\beta$ on buckling interaction curves for specially orthotropic plates $(\gamma=\delta=0)$ subjected to axial compression and subcritical transverse tension or compression loads. 


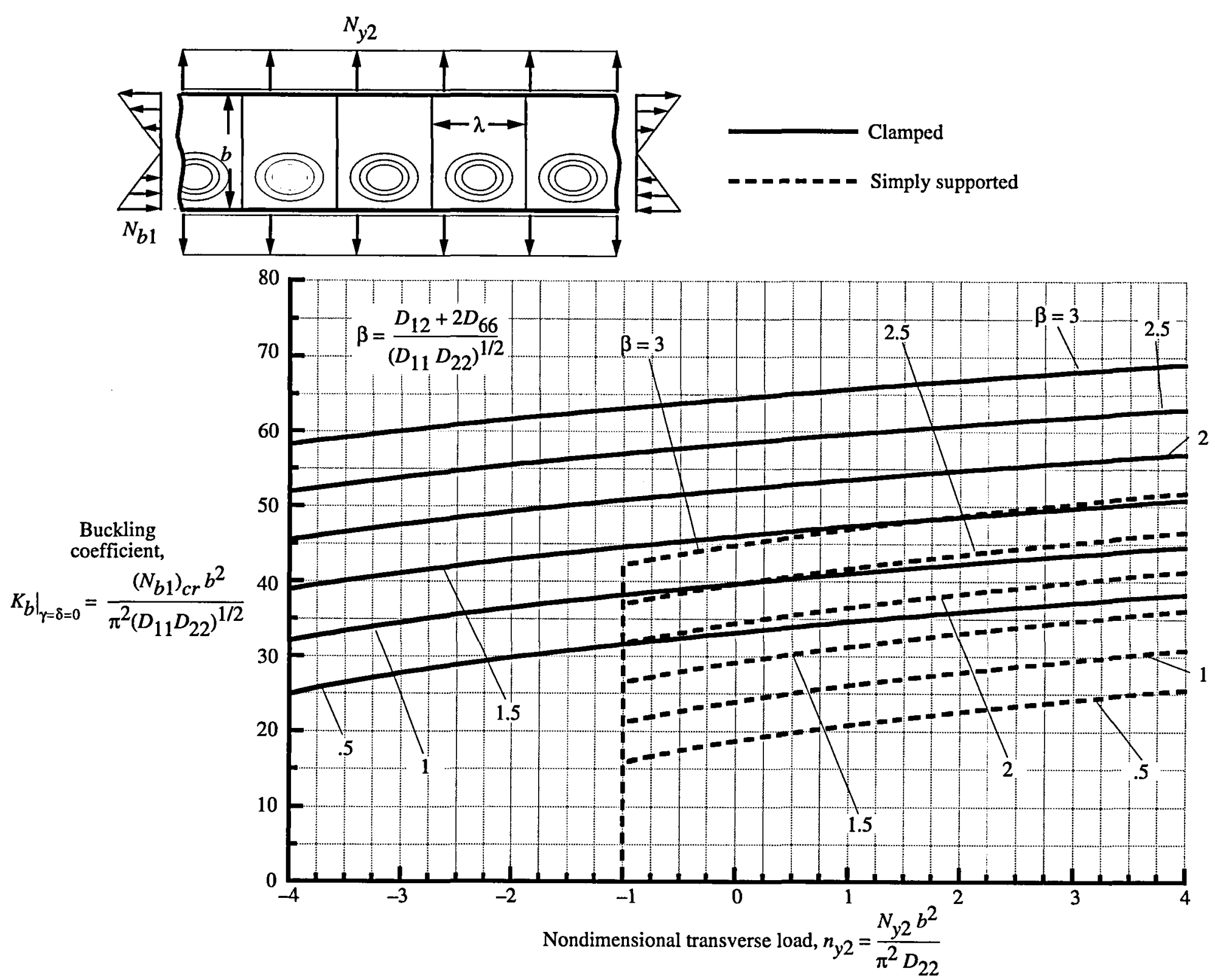

Figure 7. Effects of orthotropic parameter $\beta$ on buckling interaction curves for specially orthotropic plates $(\gamma=\delta=0)$ subjected to pure in-plane bending and subcritical transverse tension or compression loads. 


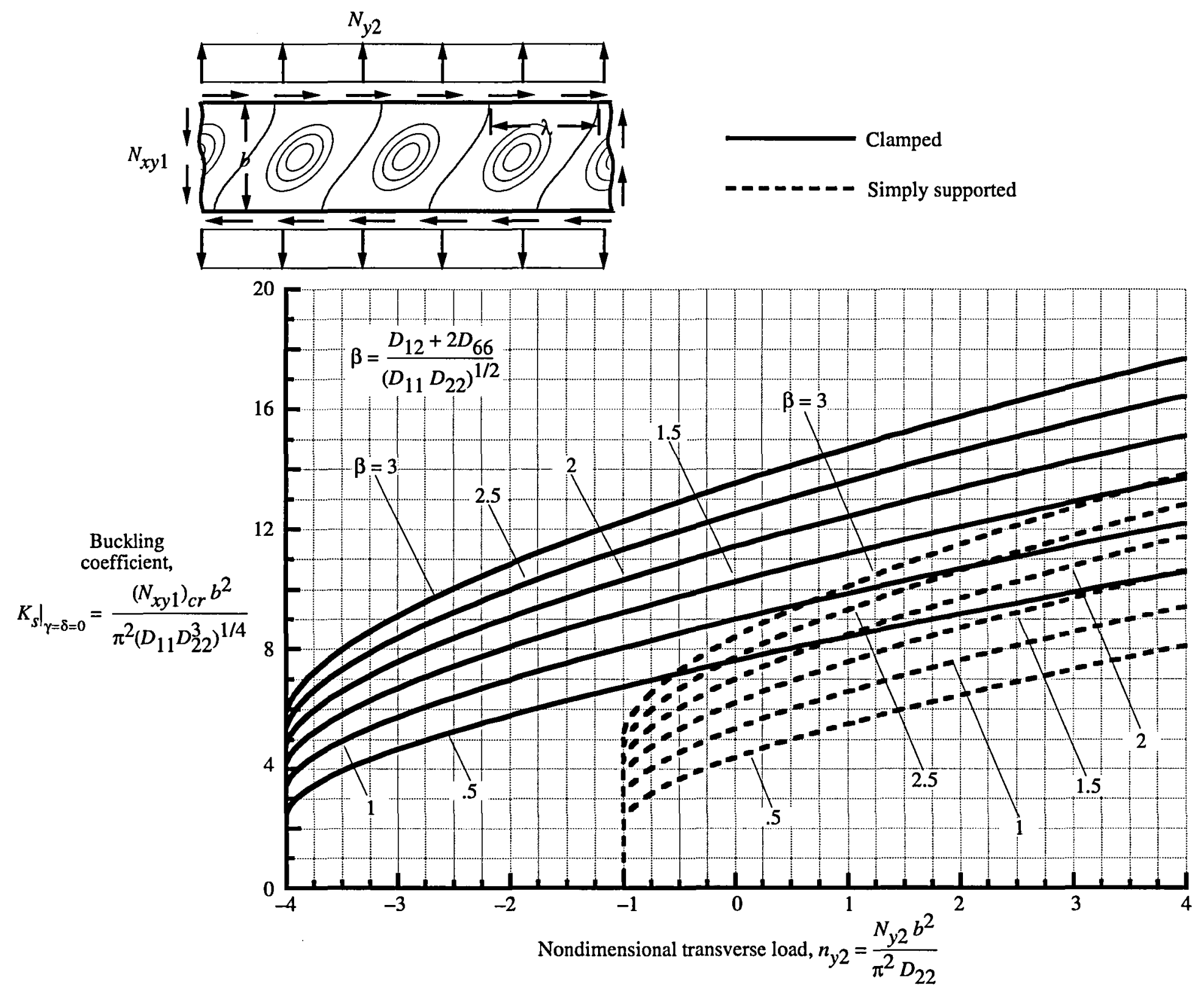

Figure 8. Effects of orthotropic parameter $\beta$ on buckling interaction curves for specially orthotropic plates $(\gamma=\delta=0)$ subjected to shear and subcritical trans- 


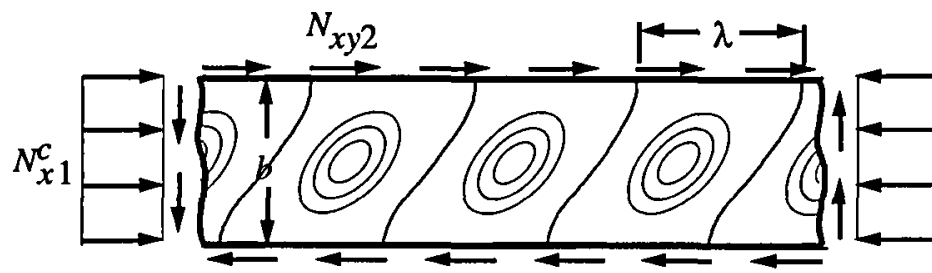

\section{Simply supported}

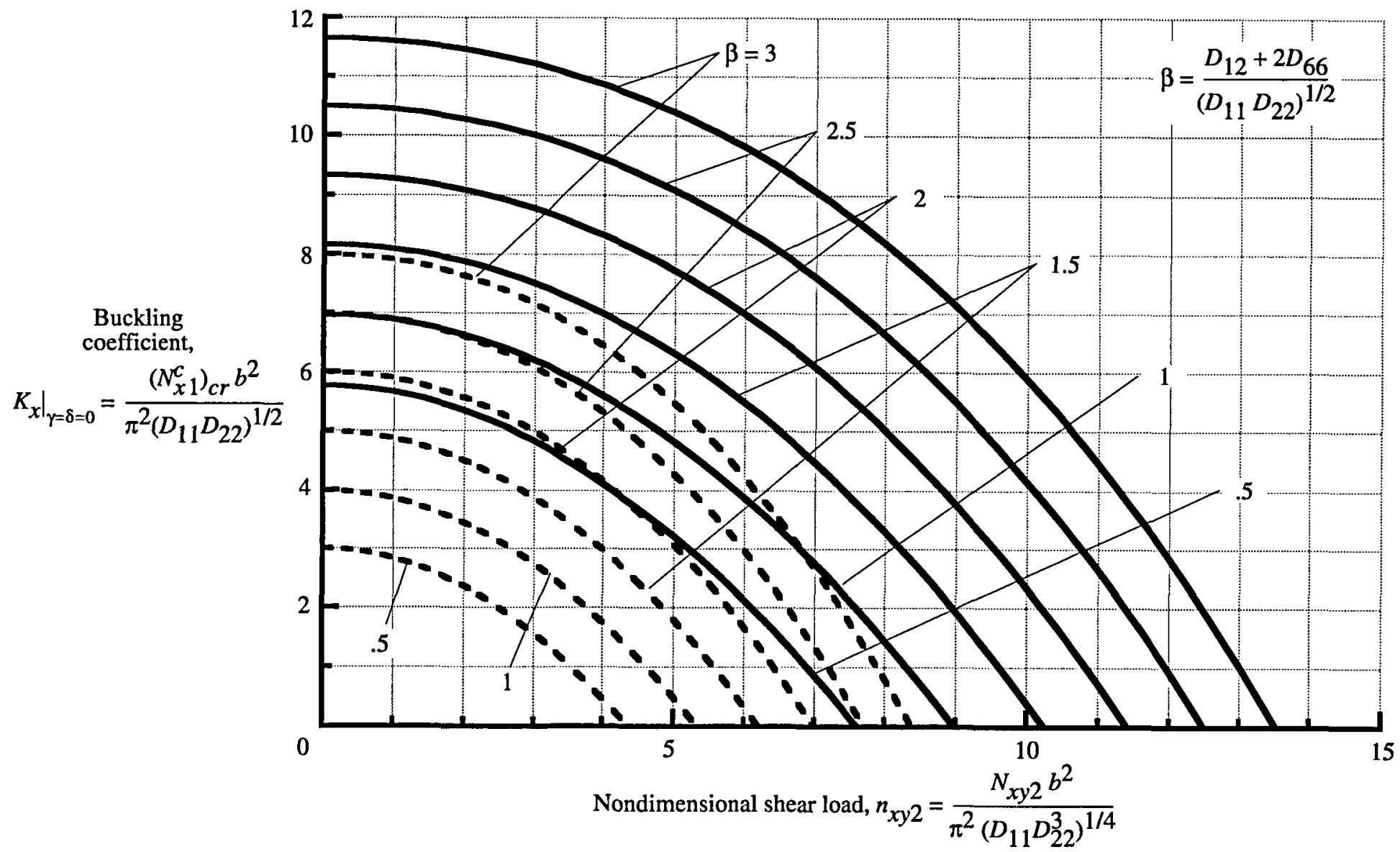

Figure 9. Effects of orthotropic parameter $\beta$ on buckling interaction curves for specially orthotropic plates $(\gamma=\delta=0)$ subjected to axial compression and subcritical shear loads. 


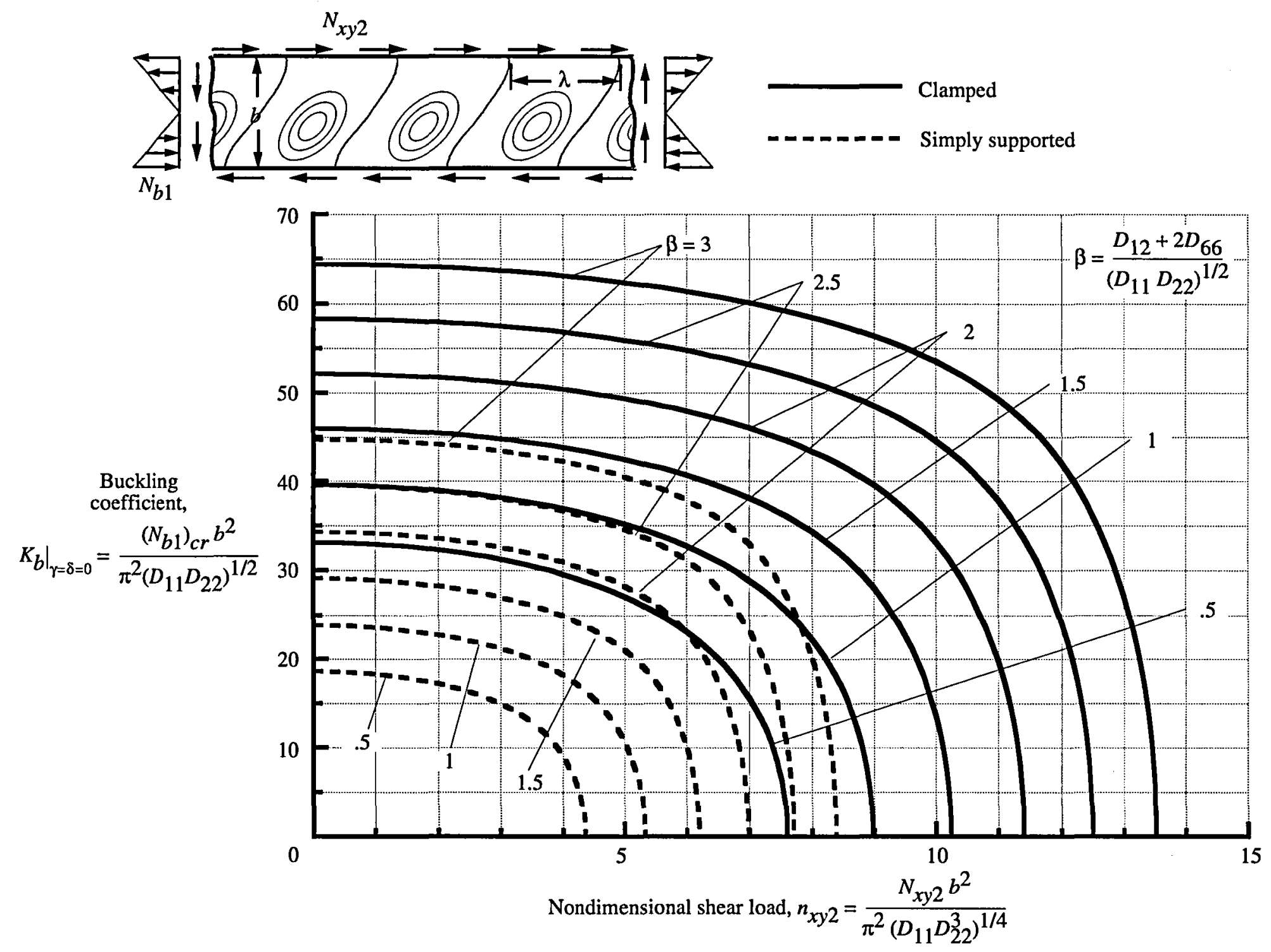

Figure 10. Effects of orthotropic parameter $\beta$ on buckling interaction curves for specially orthotropic plates $(\gamma=\delta=0)$ subjected to pure in-plane bending and subcritical shear loads. 


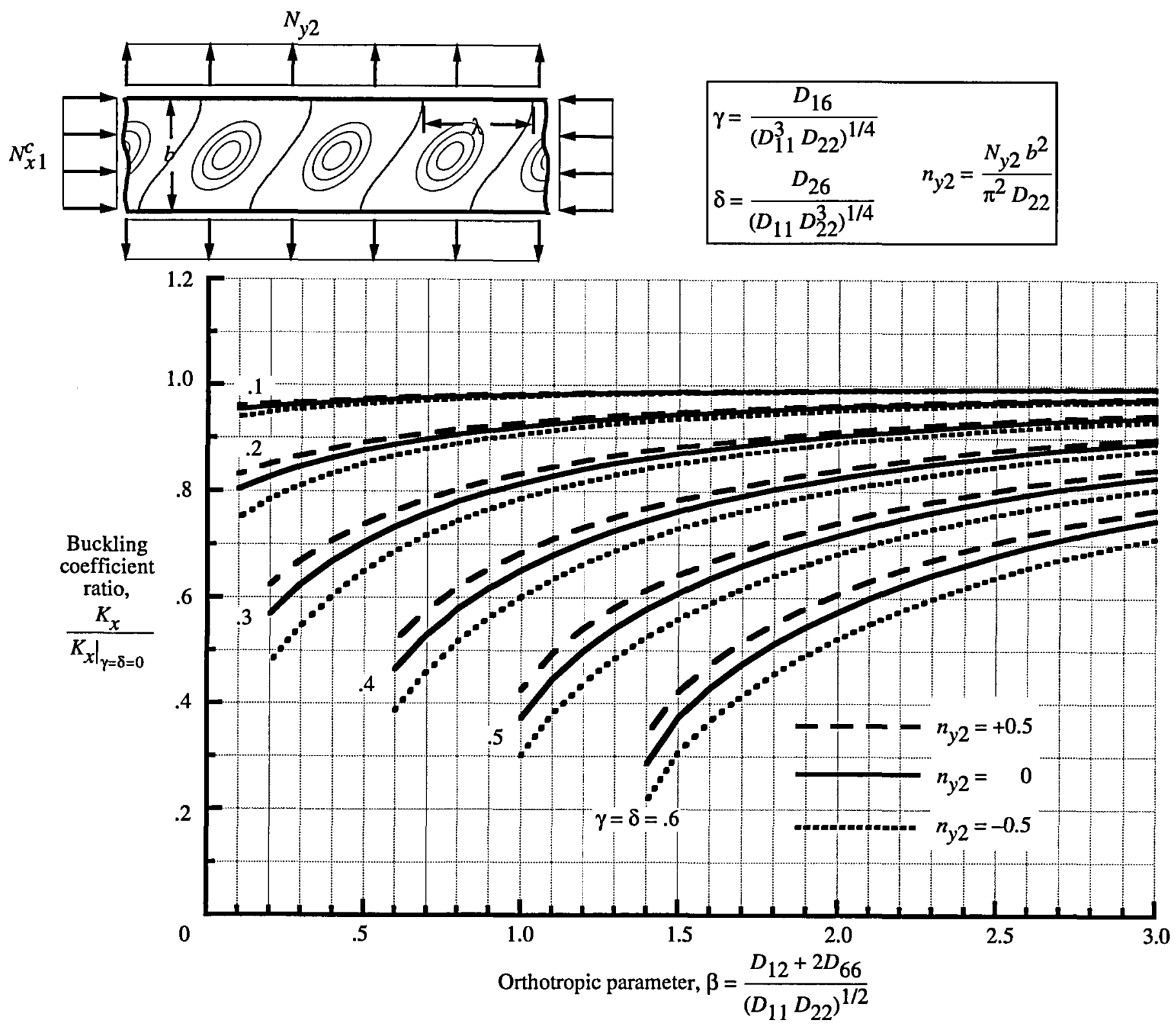

Figure 11. Effects of orthotropic parameter $\beta$ and anisotropic parameters $\gamma$ and $\delta$ on buckling coefficients for simply supported plates subjected to axial compression $\left(N_{x 1}^{c}\right)$ and subcritical transverse tension or compression $\left(N_{y 2}\right)$ loads. 


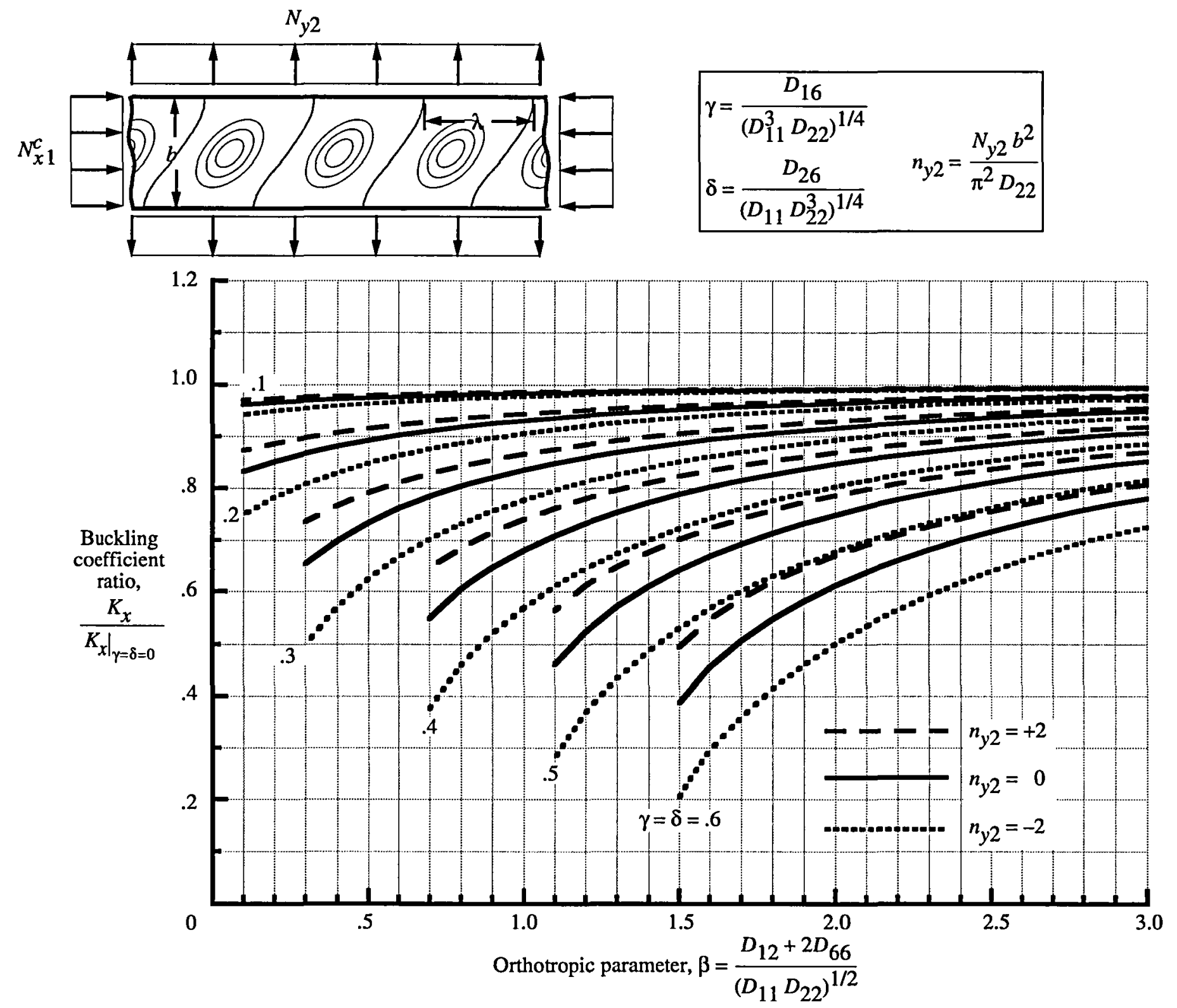

Figure 12. Effects of orthotropic parameter $\beta$ and anisotropic parameters $\gamma$ and $\delta$ on buckling coefficients for clamped plates subjected to axial compression $\left(N_{x 1}^{c}\right)$ and subcritical transverse tension or compression $\left(N_{y 2}\right)$ loads. 

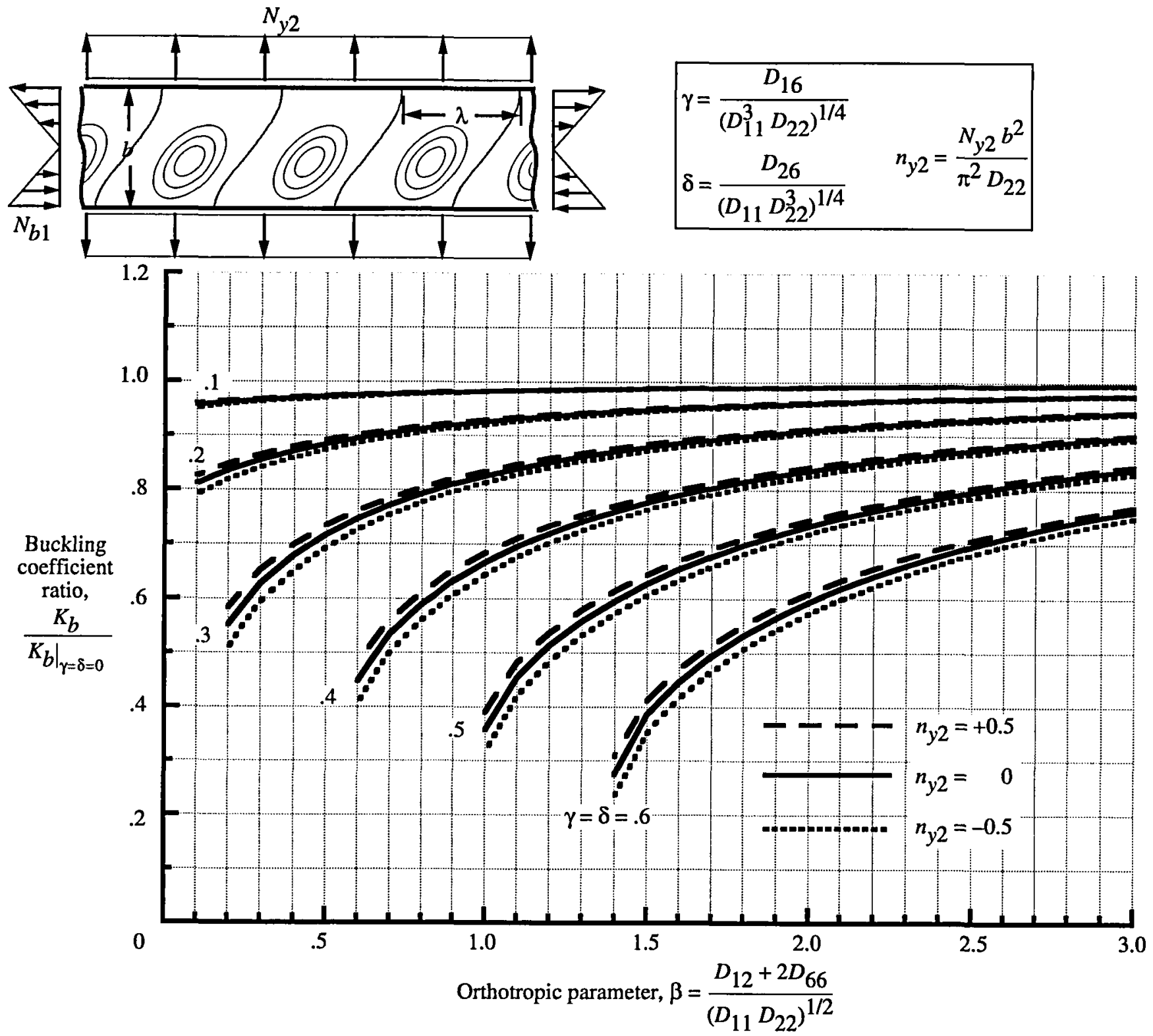

Figure 13. Effects of orthotropic parameter $\beta$ and anisotropic parameters $\gamma$ and $\delta$ on buckling coefficients for simply supported plates subjected to pure inplane bending $\left(N_{b 1}\right)$ and subcritical transverse tension or compression $\left(N_{y 2}\right)$ loads. 


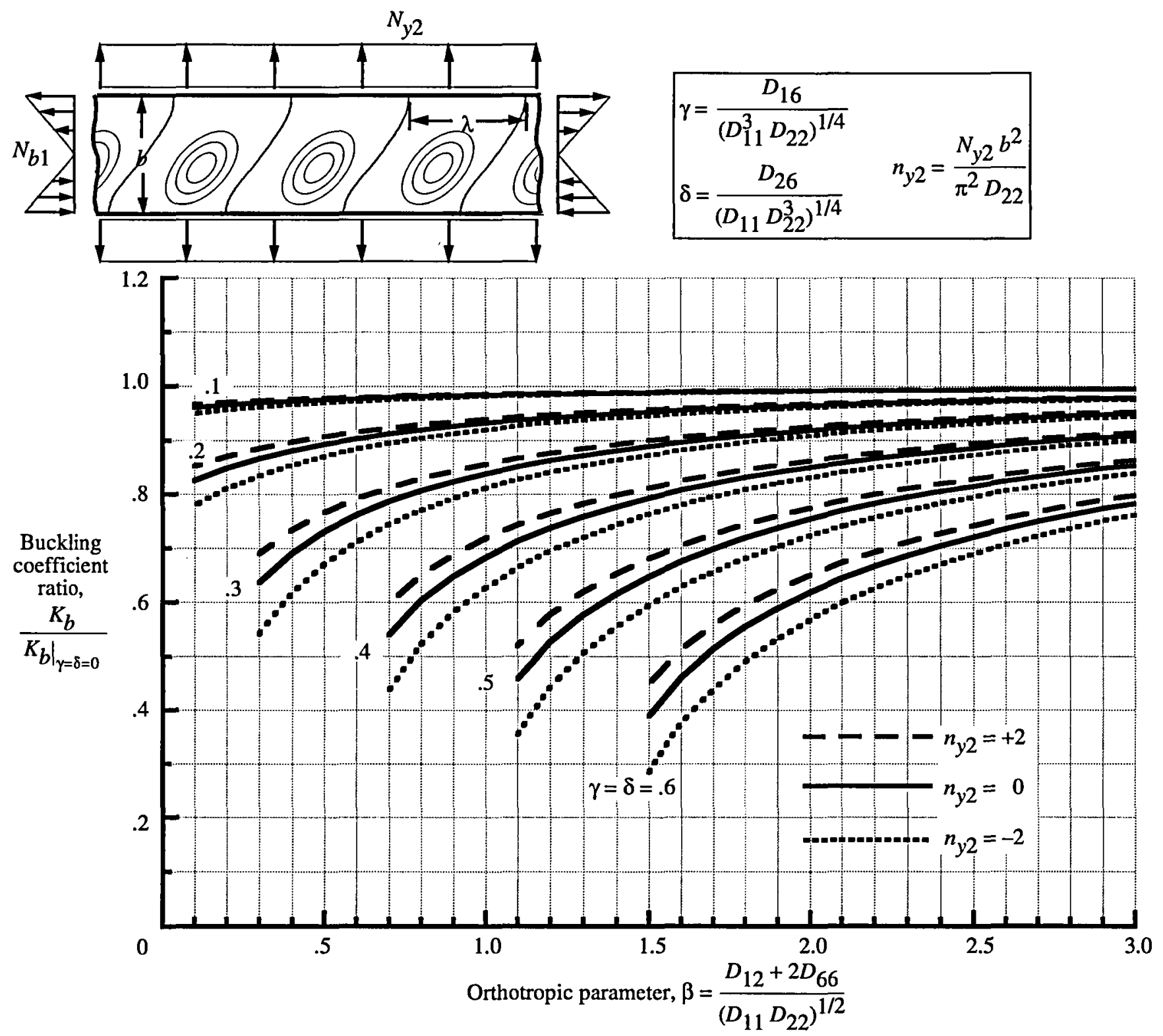

Figure 14. Effects of orthotropic parameter $\beta$ and anisotropic parameters $\gamma$ and $\delta$ on buckling coefficients for clamped plates subjected to pure in-plane bendU ing $\left(N_{b 1}\right)$ and subcritical transverse tension or compression $\left(N_{y 2}\right)$ loads. 

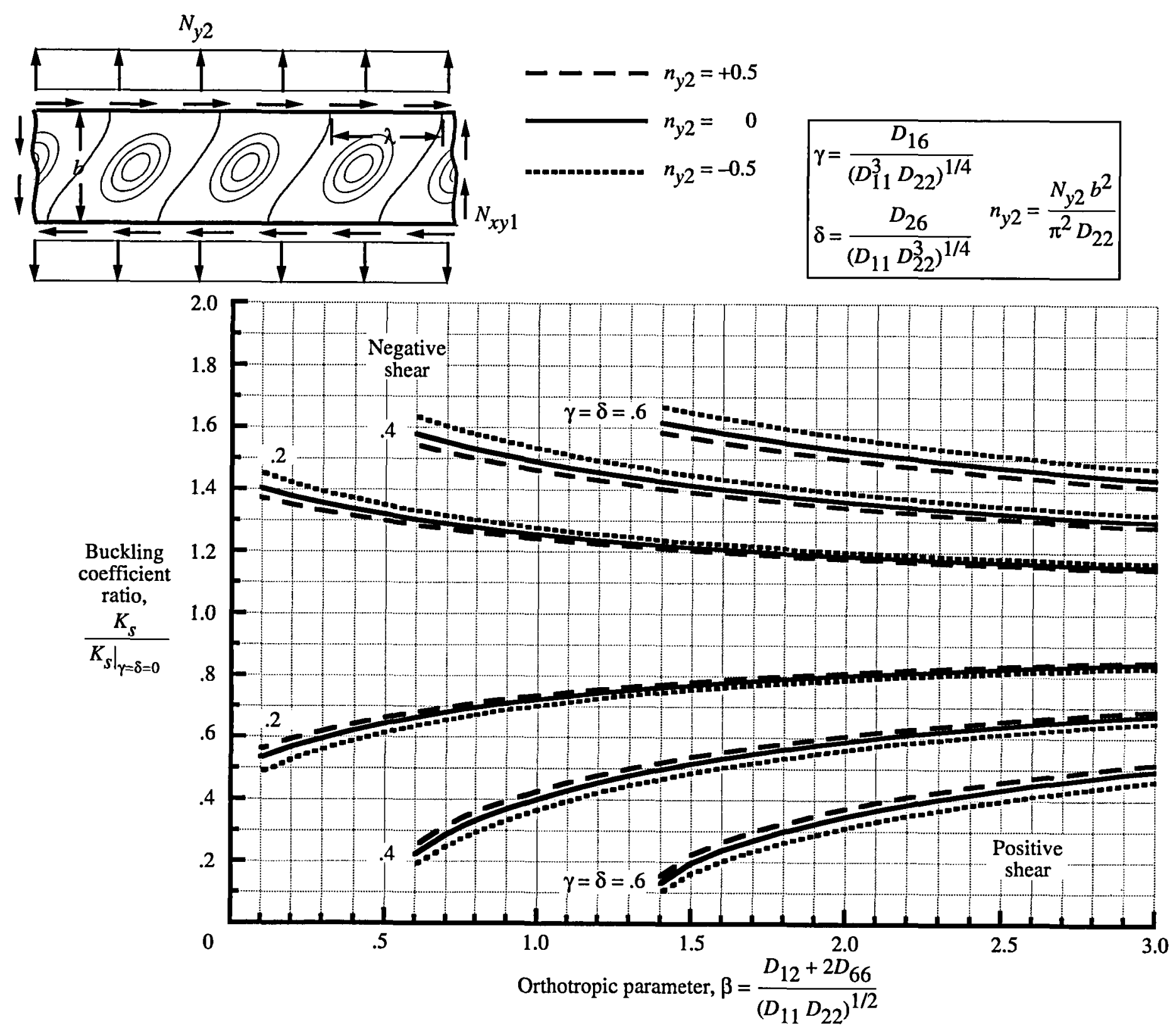

Figure 15. Effects of orthotropic parameter $\beta$ and anisotropic parameters $\gamma$ and $\delta$ on buckling coefficients for simply supported plates subjected to shear $\left(N_{x y 1}\right)$ and subcritical transverse tension or compression $\left(N_{y 2}\right)$ loads. 


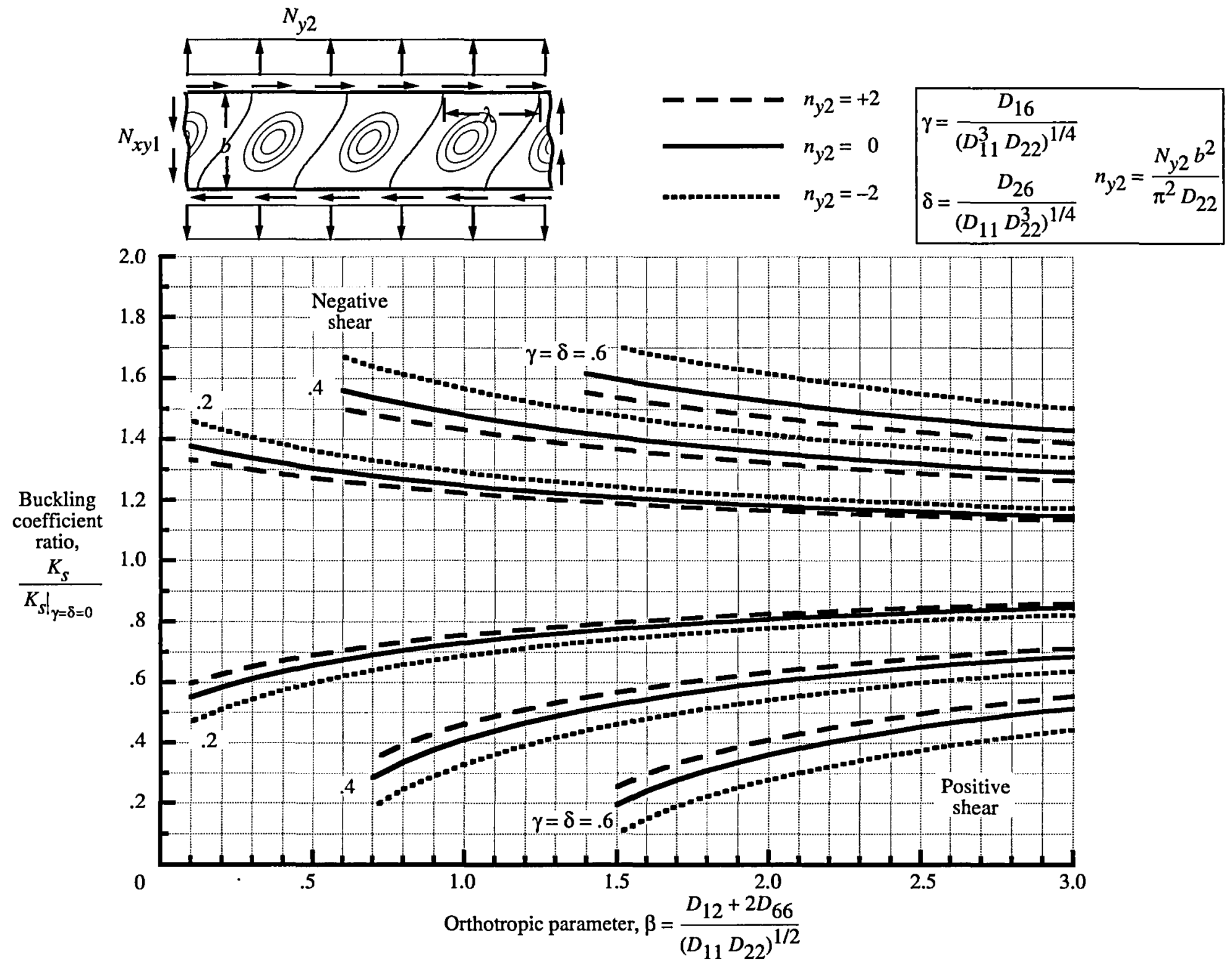

Figure 16. Effects of orthotropic parameter $\beta$ and anisotropic parameters $\gamma$ and $\delta$ on buckling coefficients for clamped plates subjected to shear $\left(N_{x y 1}\right)$ and subcritical transverse tension or compression $\left(N_{y 2}\right)$ loads. 

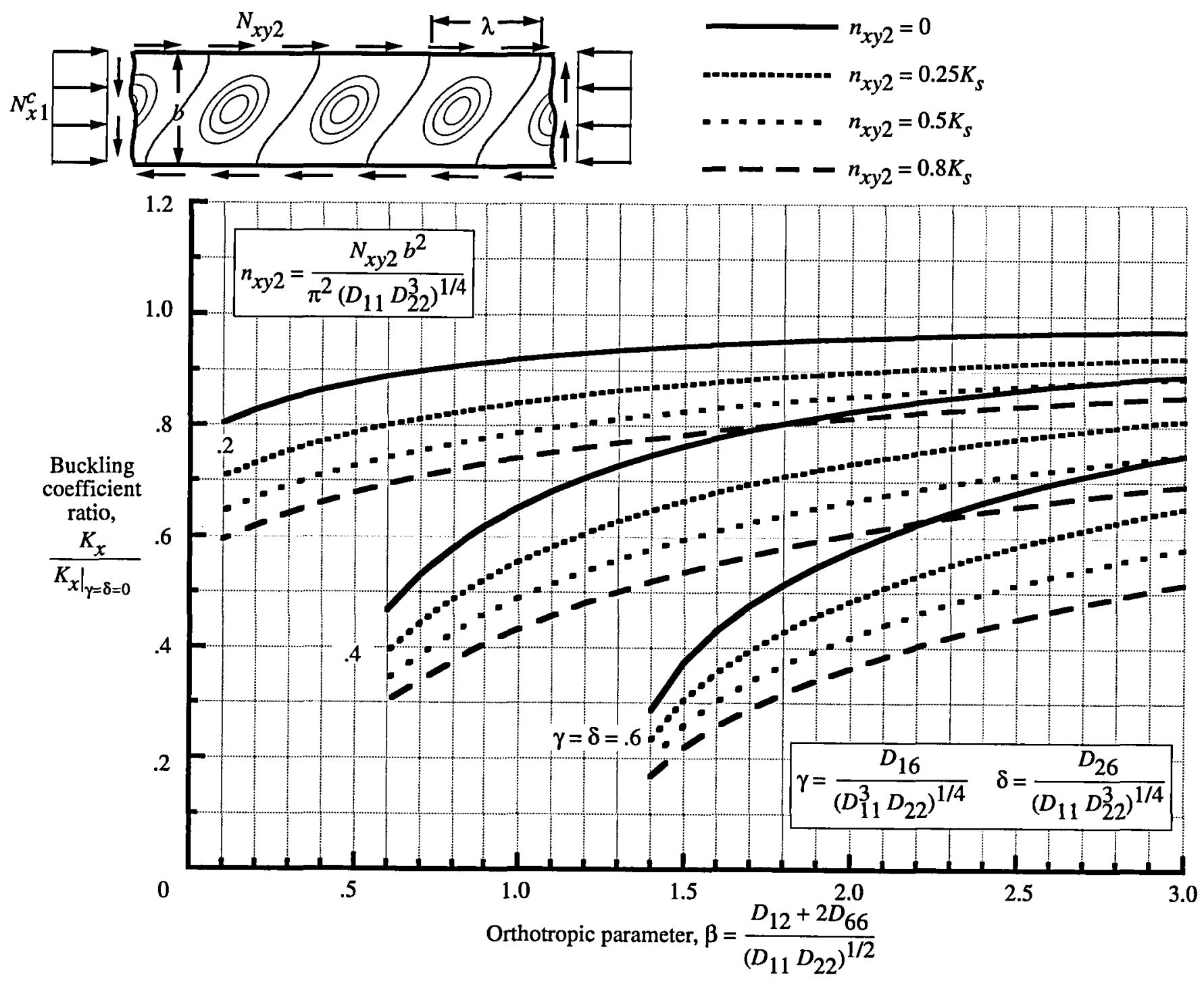

Figure 17. Effects of orthotropic parameter $\beta$ and anisotropic parameters $\gamma$ and $\delta$ on buckling coefficients for simply supported plates subjected to axial compression $\left(N_{x 1}^{c}\right)$ and positive subcritical shear $\left(n_{x y 2}\right)$ loads. 

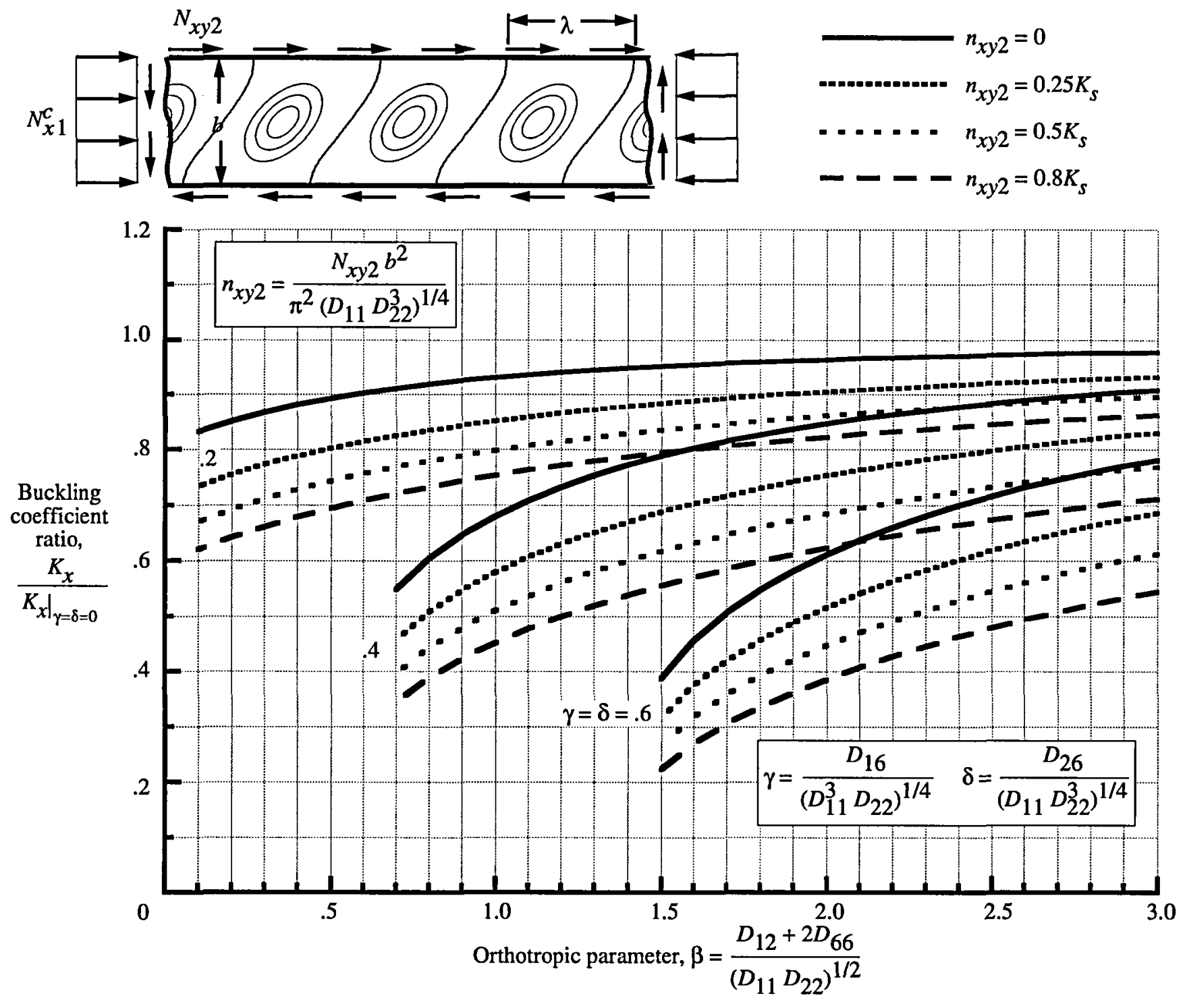

Figure 18. Effects of orthotropic parameter $\beta$ and anisotropic parameters $\gamma$ and $\delta$ on buckling coefficients for clamped plates subjected to axial compression $\left(N_{x 1}^{c}\right)$ and positive subcritical shear $\left(n_{x y 2}\right)$ loads. 

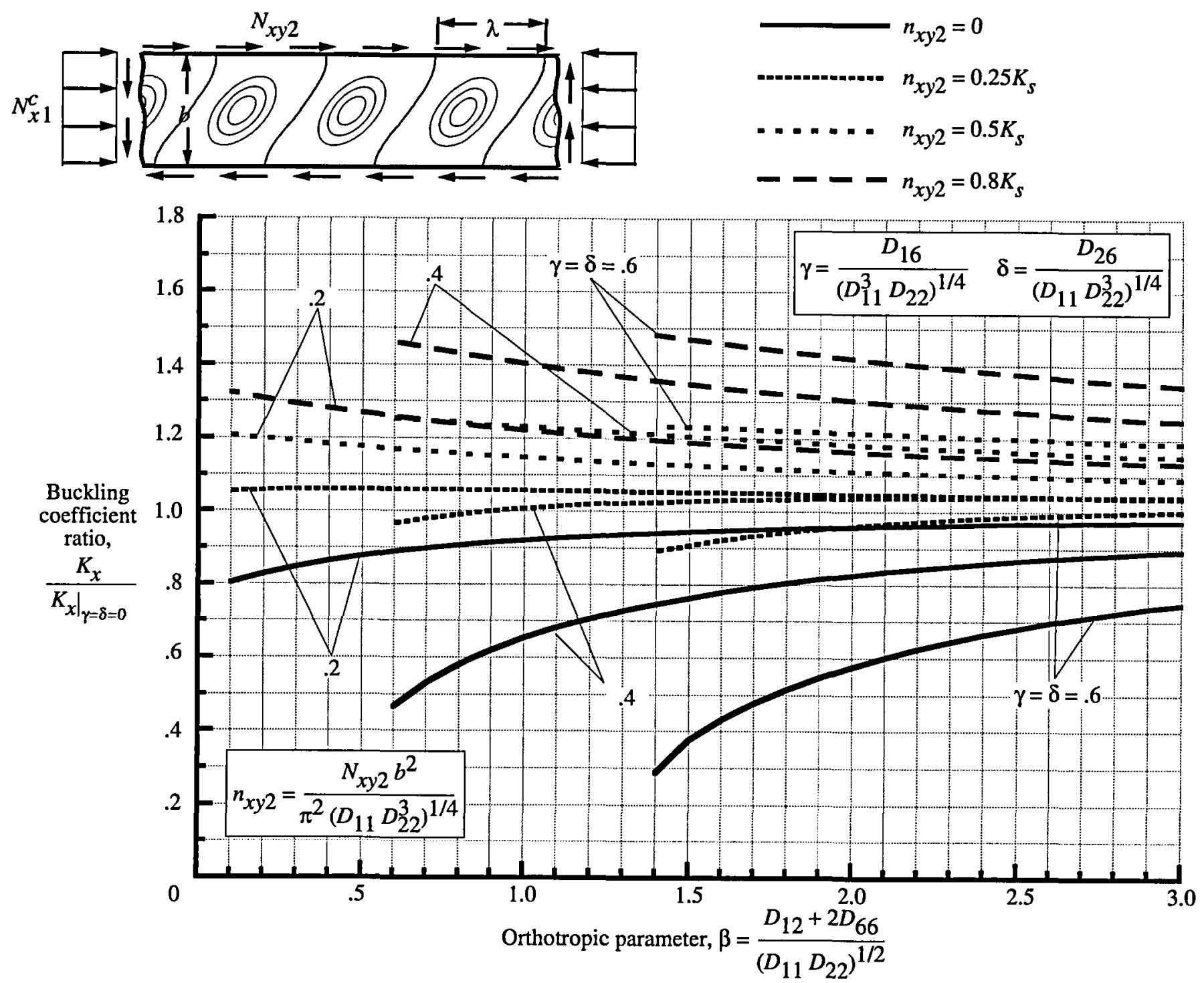

Figure 19. Effects of orthotropic parameter $\beta$ and anisotropic parameters $\gamma$ and $\delta$ on buckling coefficients for simply supported plates subjected to axial compression $\left(N_{x 1}^{c}\right)$ and negative subcritical shear $\left(n_{x y 2}\right)$ loads. 

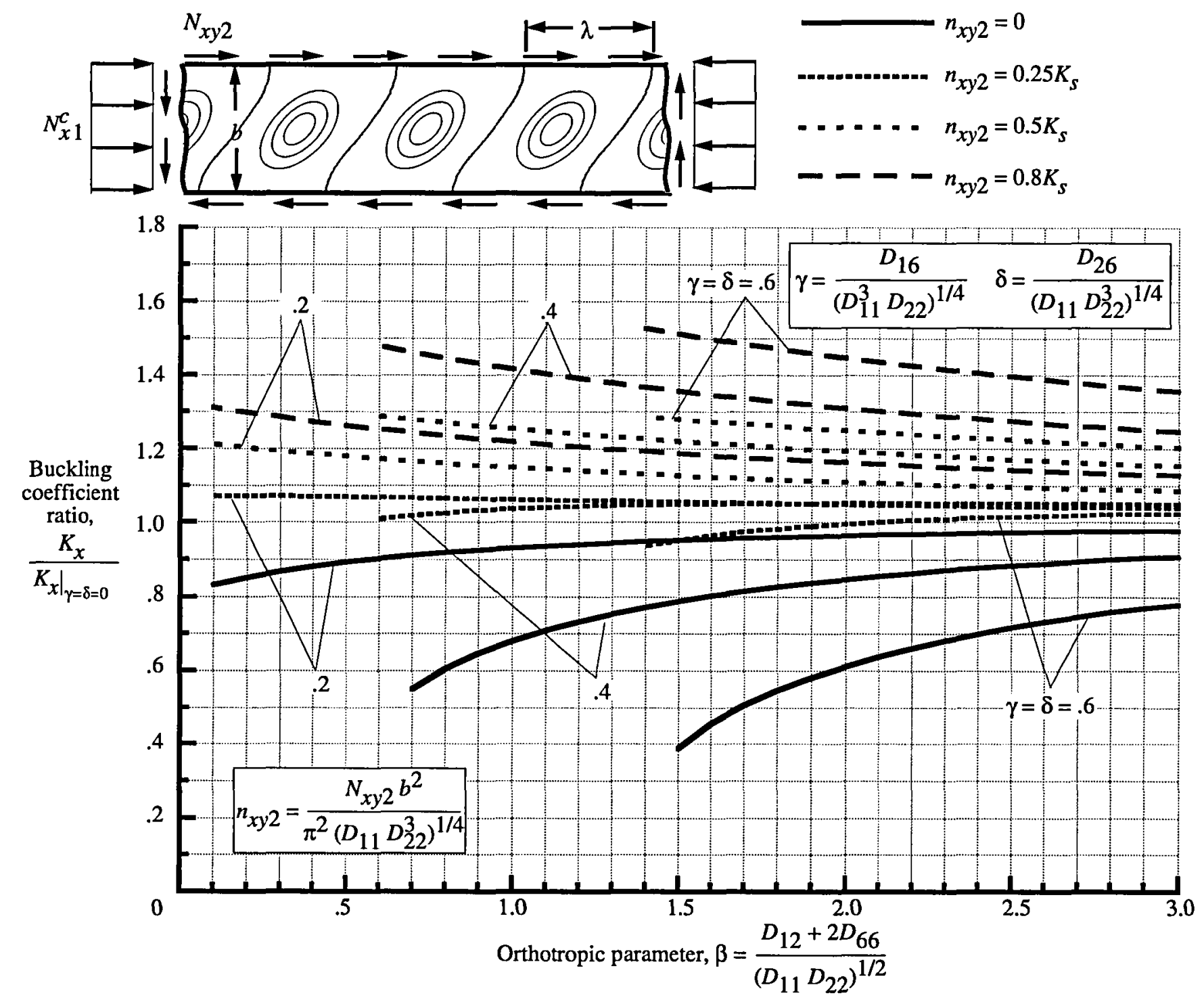

Figure 20. Effects of orthotropic parameter $\beta$ and anisotropic parameters $\gamma$ and $\delta$ on buckling coefficients for clamped plates subjected to axial compression $\left(N_{x 1}^{c}\right)$ and negative subcritical shear $\left(n_{x y 2}\right)$ loads. 


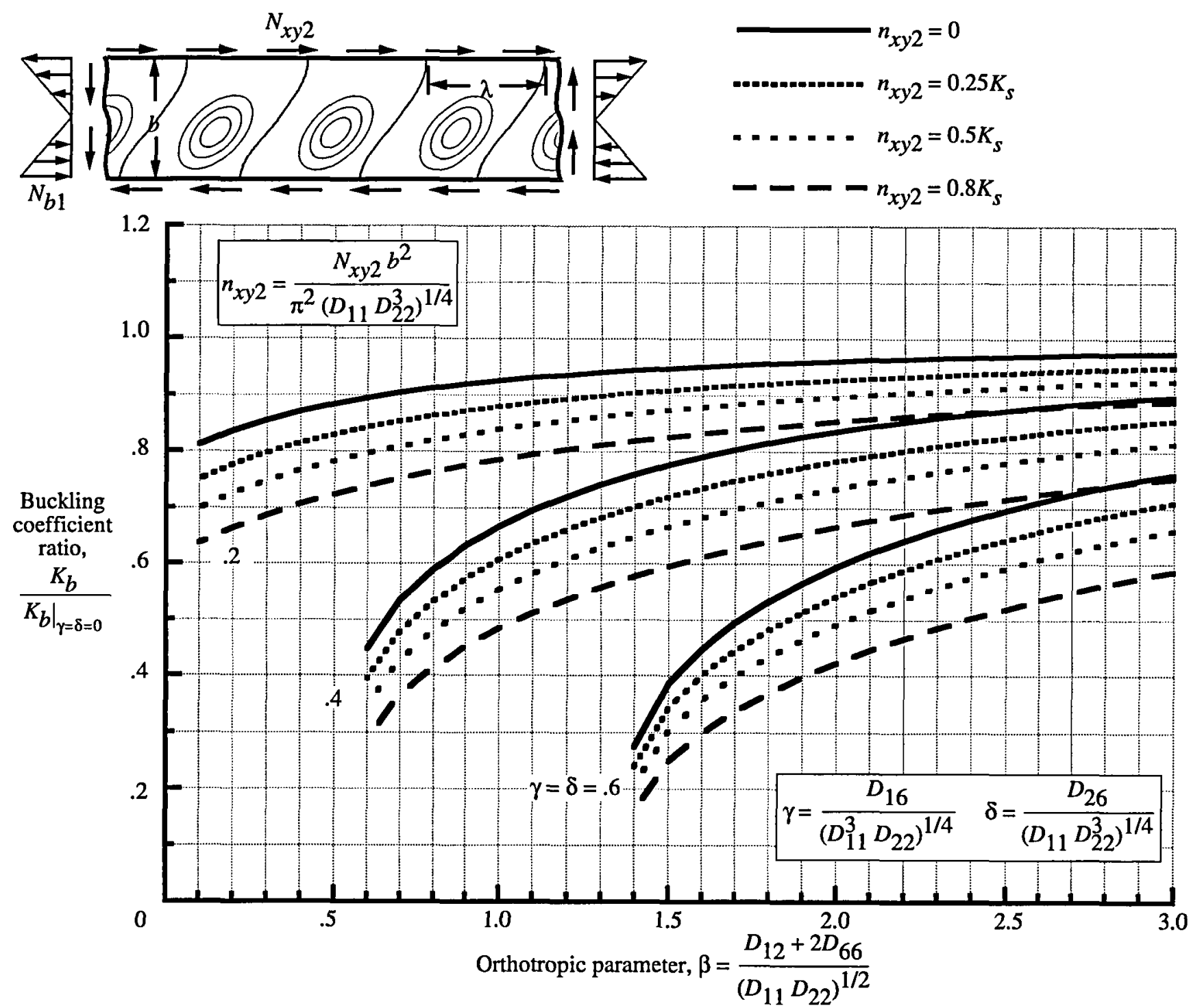

Figure 21. Effects of orthotropic parameter $\beta$ and anisotropic parameters $\gamma$ and $\delta$ on buckling coefficients for simply supported plates subjected to pure inplane bending $\left(N_{b 1}\right)$ and positive subcritical shear $\left(n_{x y 2}\right)$ loads. 


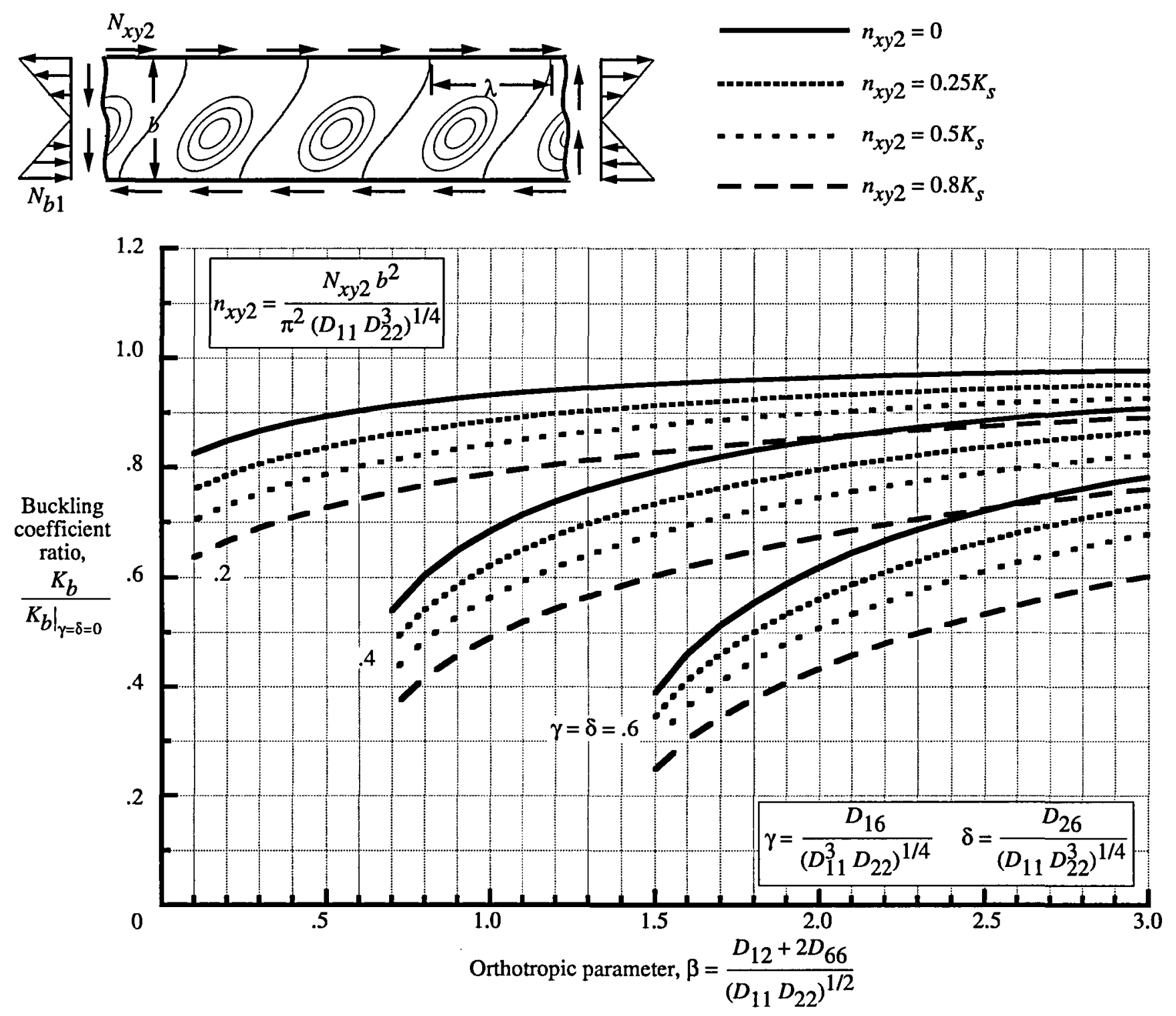

Figure 22. Effects of orthotropic parameter $\beta$ and anisotropic parameters $\gamma$ and $\delta$ on buckling coefficients for clamped plates subjected to pure in-plane bending $\left(N_{b 1}\right)$ and positive subcritical shear $\left(n_{x y 2}\right)$ loads. 


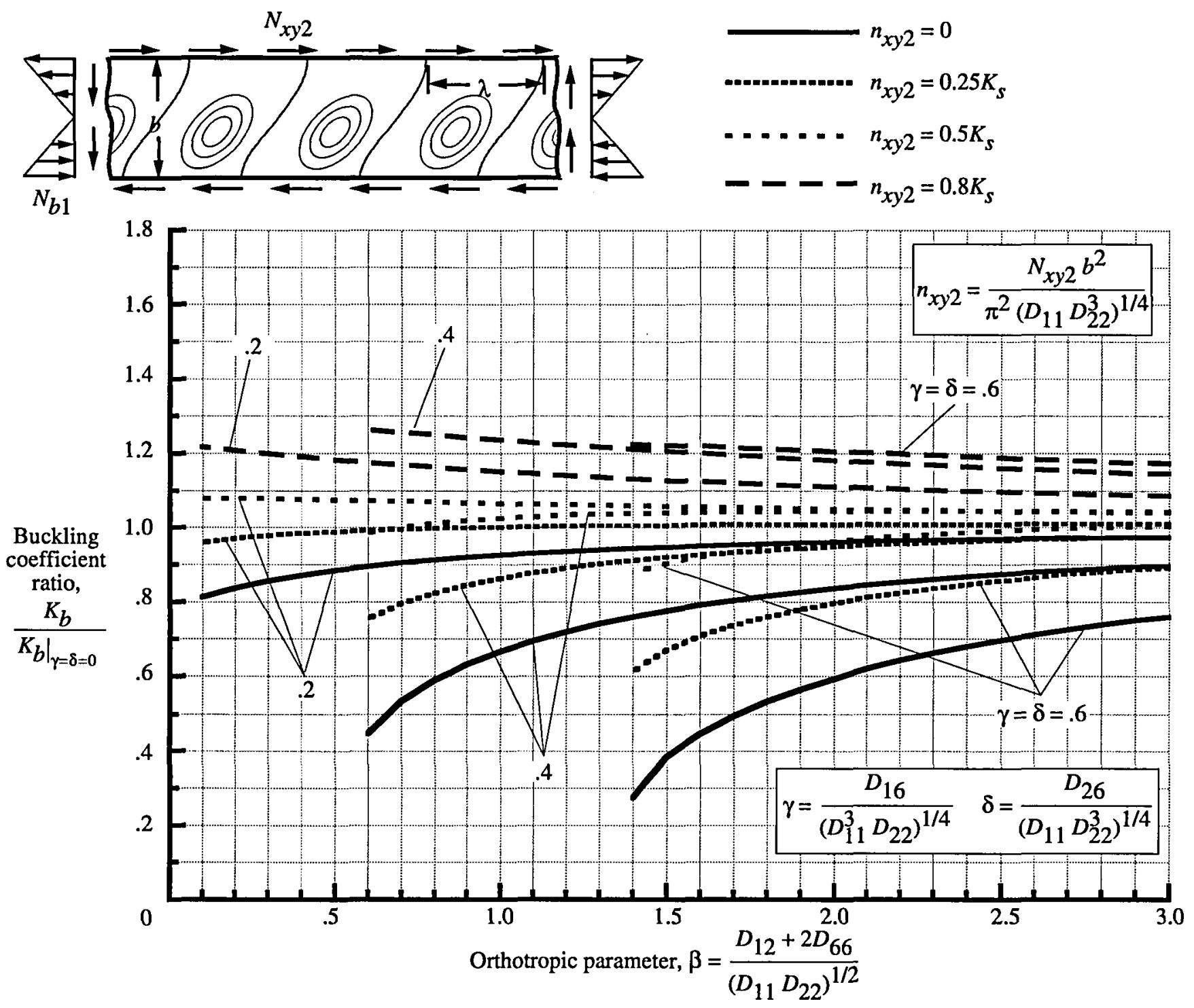

Figure 23. Effects of orthotropic parameter $\beta$ and anisotropic parameters $\gamma$ and $\delta$ on buckling coefficients for simply supported plates subjected to pure inplane bending $\left(N_{b 1}\right)$ and negative subcritical shear $\left(n_{x y 2}\right)$ loads. 


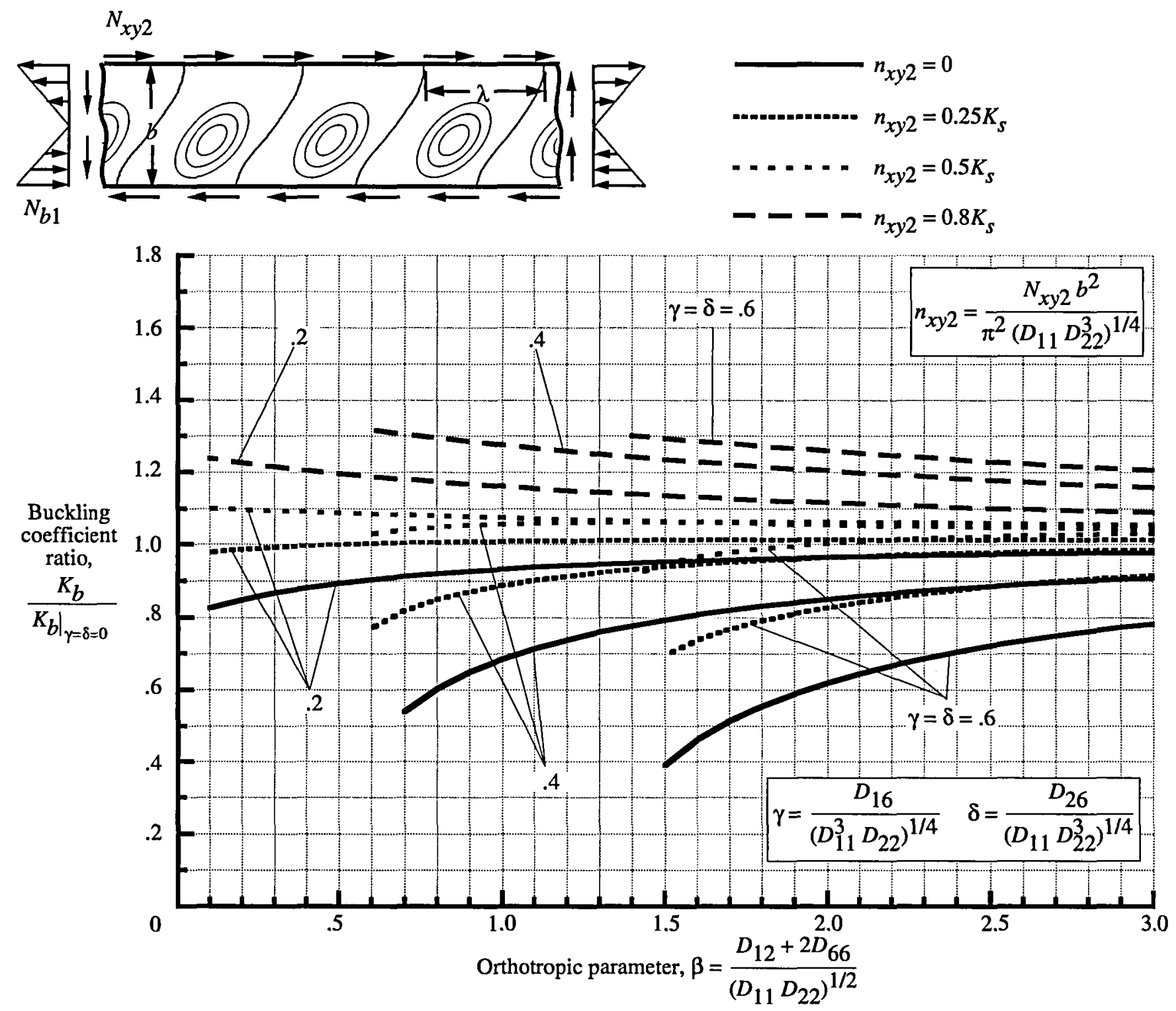

Figure 24. Effects of orthotropic parameter $\beta$ and anisotropic parameters $\gamma$ and $\delta$ on buckling coefficients for clamped plates subjected to pure in-plane bending $\left(N_{b 1}\right)$ and negative subcritical shear $\left(n_{x y 2}\right)$ loads. 

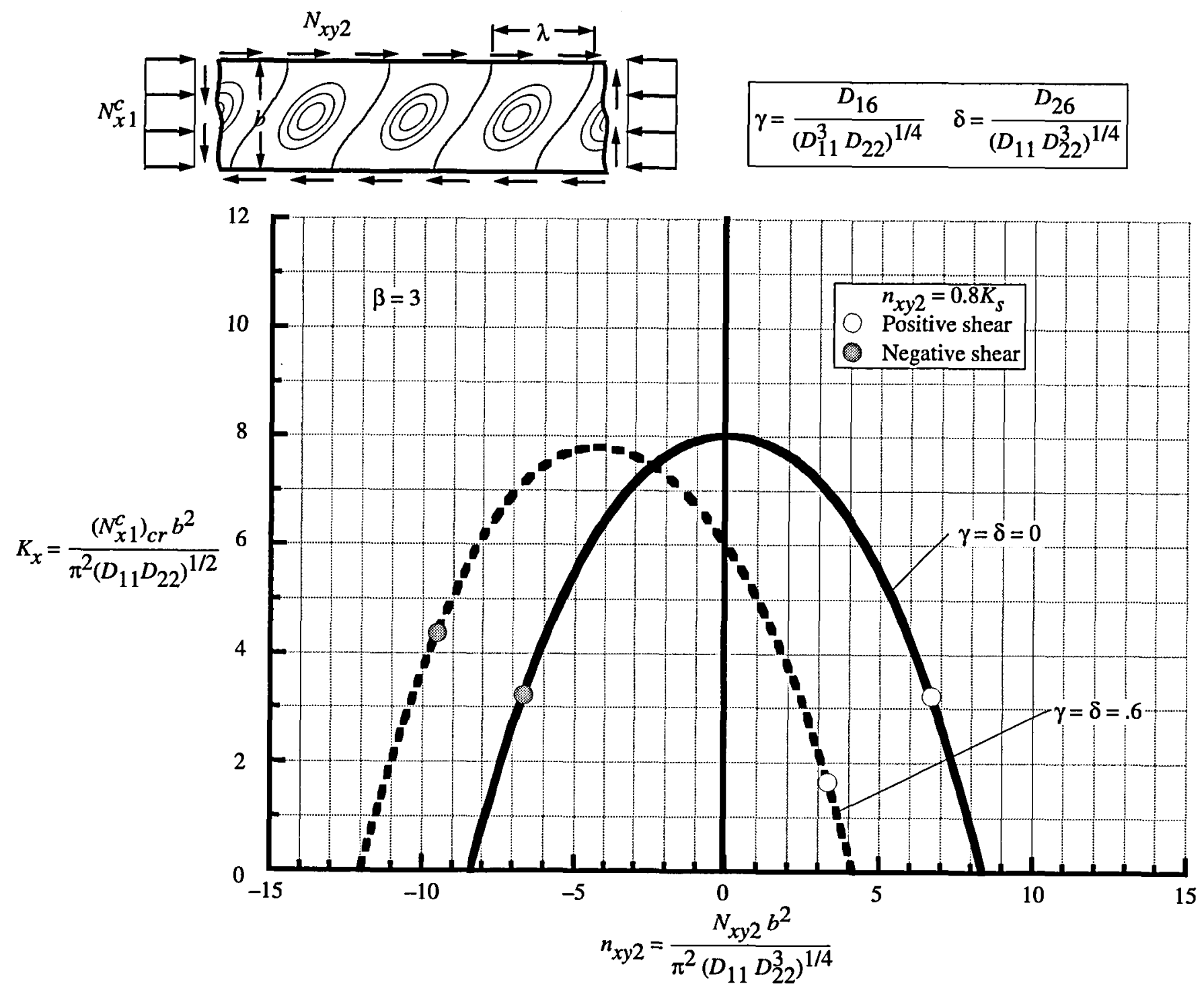

Figure 25. Buckling interaction curves for simply supported plates subjected to axial compression $\left(N_{x 1}^{c}\right)$ and subcritical shear $\left(n_{x y 2}\right)$ loads $(\beta=3, \gamma=\delta=0$, and 0.6). 


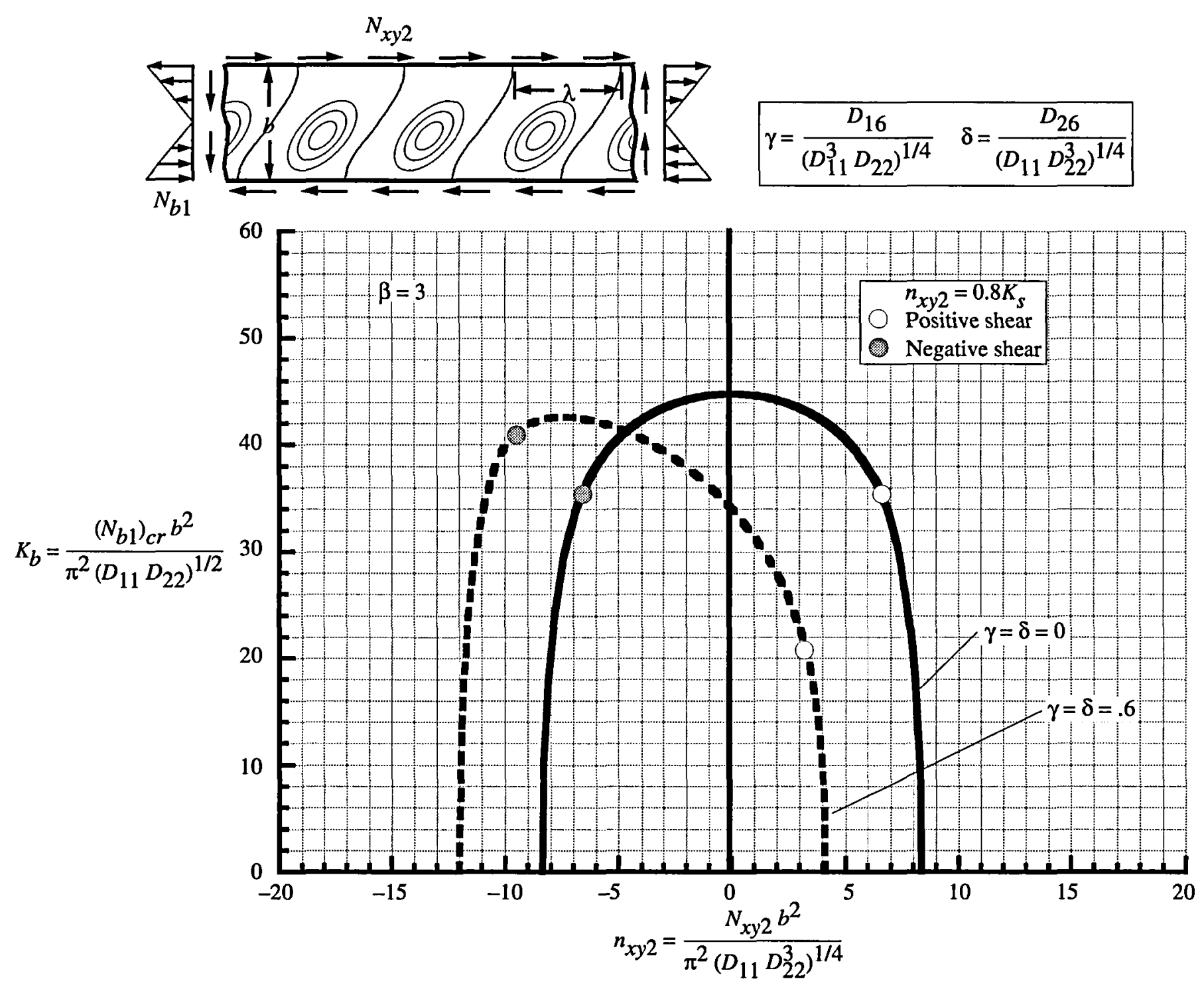

Figure 26. Buckling interaction curves for simply supported plates subjected to pure in-plane bending $\left(N_{b 1}\right)$ and subcritical shear $\left(n_{x y 2}\right)$ loads $(\beta=3$, $\gamma=\delta=0$, and 0.6 ). 


\begin{tabular}{|c|c|c|c|c|}
\hline \multicolumn{3}{|c|}{ REPORT DOCUMENTATION PAGE } & \multicolumn{2}{|r|}{$\begin{array}{l}\text { Form Approved } \\
\text { OMB No. 0704-0188 }\end{array}$} \\
\hline \multicolumn{5}{|c|}{ 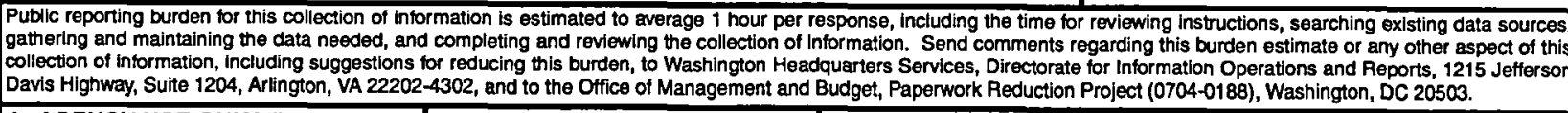 } \\
\hline 1. AGENCY USE ONLY (Leave blank) & $\begin{array}{l}\text { 2. REPORT DATE } \\
\text { November } 1995\end{array}$ & \multicolumn{3}{|c|}{$\begin{array}{l}\text { 3. REPORT TYPE AND DATES COVERED } \\
\text { Technical Paper }\end{array}$} \\
\hline \multicolumn{3}{|c|}{$\begin{array}{l}\text { 4. TTLE AND SUBTITLE } \\
\text { Buckling Behavior of Long Anisotropic Plates Subjected to Combined Loads }\end{array}$} & \multirow{2}{*}{\multicolumn{2}{|c|}{$\begin{array}{l}\text { 5. FUNDING NUMBERS } \\
\text { WU 505-63-50-08 }\end{array}$}} \\
\hline \multicolumn{3}{|l|}{$\begin{array}{l}\text { 6. AUTHOA(S) } \\
\text { Michael P. Nemeth }\end{array}$} & & \\
\hline \multicolumn{3}{|c|}{$\begin{array}{l}\text { NASA Langley Research Center } \\
\text { Hampton, VA 23681-0001 }\end{array}$} & \multicolumn{2}{|c|}{$\begin{array}{l}\text { 8. PERFORMING ORGANIZATION } \\
\text { REPORT NUMBER } \\
\text { L-17504 }\end{array}$} \\
\hline $\begin{array}{l}\text { 9. SPONSOAING/MONTORING AGEN } \\
\text { National Aeronautics and Sp: } \\
\text { Washington, DC 20546-0001 }\end{array}$ & $\begin{array}{l}\text { CY NAME(S) AND ADDRESS(ES) } \\
\text { ace Administration }\end{array}$ & & \multicolumn{2}{|c|}{$\begin{array}{l}\text { 10. SPONSORING/MONITORING } \\
\text { AGENCY REPORT NUMBER } \\
\text { NASA TP-3568 }\end{array}$} \\
\hline \multicolumn{5}{|c|}{$\begin{array}{l}\text { 11. SUPPLEMENTARY NOTES } \\
\text { Presented at the 36th AIAA/ASME/ASCE/ASC/AHS Structures, Structural Dynamics, and Materials Conference, } \\
\text { April 10-12, 1995, New Orleans, LA. }\end{array}$} \\
\hline \multicolumn{3}{|c|}{$\begin{array}{l}\text { 12a. DISTRIBUTION/AVAILABILTT STATEMENT } \\
\text { Unclassified-Unlimited } \\
\text { Subject Category } 39 \\
\text { Availability: NASA CASI (301) 621-0390 }\end{array}$} & \multicolumn{2}{|c|}{ 12b. DISTRIBUTION CODE } \\
\hline \multicolumn{5}{|c|}{$\begin{array}{l}\text { 13. ABSTRACT (Maximum } 200 \text { words) } \\
\text { A parametric study is presented of the buckling behavior of infinitely long symmetrically laminated anisotropic } \\
\text { plates subjected to combined loads. The study focuses on the interaction of a subcritical (stable) secondary loading } \\
\text { state of constant magnitude and a primary destabilizing load that is increased in magnitude until buckling occurs. } \\
\text { The loads considered in this report are uniform axial compression, pure in-plane bending, transverse tension and } \\
\text { compression, and shear. Results are presented that were obtained by using a special purpose nondimensional analy- } \\
\text { sis that is well suited for parametric studies of clamped and simply supported plates. In particular, results are pre- } \\
\text { sented for a }[ \pm 45]_{s} \text { graphite-epoxy laminate that is highly anisotropic and representative of a laminate used for } \\
\text { spacecraft applications. In addition, generic buckling-design charts are presented for a wide range of nondimen- } \\
\text { sional parameters that are applicable to a broad class of laminate constructions. These results show the general } \\
\text { behavioral trends of specially orthotropic plates and the effects of flexural anisotropy on plates subjected to various } \\
\text { combined loading conditions. An important finding of the present study is that the effects of flexural anisotropy on } \\
\text { the buckling resistance of a plate can be significantly more important for plates subjected to combined loads than } \\
\text { for plates subjected to single-component loads. }\end{array}$} \\
\hline \multirow{2}{*}{\multicolumn{4}{|c|}{$\begin{array}{l}\text { 14. SUBJECT TERMS } \\
\text { Buckling; Combined loads; Anisotropy; Flat plates; Composite plates }\end{array}$}} & $\begin{array}{c}\text { 15. NUMBER OF PAGES } \\
38 \\
\end{array}$ \\
\hline & & & & $\begin{array}{r}\text { 16. PRICE CODE } \\
\text { A03 }\end{array}$ \\
\hline $\begin{array}{l}\text { 17. SECURITY CLASSIFICATION } \\
\text { OF REPORT } \\
\text { Unclassified }\end{array}$ & $\begin{array}{l}\text { 18. SECURTYY CLASSIFICATION } \\
\text { OF THIS PAGE } \\
\text { Unclassified }\end{array}$ & $\begin{array}{l}\text { 19. SECURTY CLASSIF } \\
\text { OF ABSTRACT } \\
\text { Unclassified }\end{array}$ & ICATION & $\begin{array}{l}\text { 20. LIMITATION } \\
\text { OF ABSTRACT }\end{array}$ \\
\hline NSN 7540-01-280-5500 & & & & $\begin{array}{l}\text { Standard Form } 298 \text { (Rev. 2-89) } \\
\text { Prescribed by ANSI Std. Z39-18 } \\
298-102\end{array}$ \\
\hline
\end{tabular}


